\title{
Fiber orientation interpolation for the multiscale analysis of short fiber reinforced composite parts
}

\author{
Jonathan Köbler $^{1}$ • Matti Schneider ${ }^{1}$ - Felix Ospald ${ }^{2}$ - Heiko Andrä ${ }^{1}$. \\ Ralf Müller ${ }^{3}$
}

Received: 26 April 2017 / Accepted: 21 June 2017 / Published online: 13 April 2018

(C) The Author(s) 2018

\begin{abstract}
For short fiber reinforced plastic parts the local fiber orientation has a strong influence on the mechanical properties. To enable multiscale computations using surrogate models we advocate a two-step identification strategy. Firstly, for a number of sample orientations an effective model is derived by numerical methods available in the literature. Secondly, to cover a general orientation state, these effective models are interpolated. In this article we develop a novel and effective strategy to carry out this interpolation. Firstly, taking into account symmetry arguments, we reduce the fiber orientation phase space to a triangle in $\mathbb{R}^{2}$. For an associated triangulation of this triangle we furnish each node with an surrogate model. Then, we use linear interpolation on the fiber orientation triangle to equip each fiber orientation state with an effective stress. The proposed approach is quite general, and works for any physically nonlinear constitutive law on the micro-scale, as long as surrogate models for single fiber orientation states can be extracted. To demonstrate the capabilities of our scheme we study the viscoelastic creep behavior of short glass fiber reinforced PA66, and use Schapery's collocation method together with FFT-based computational homogenization to derive single orientation
\end{abstract}

Jonathan Köbler

jonathan.koebler@itwm.fraunhofer.de

1 Department of Flow and Materials Simulation, Fraunhofer ITWM, Kaiserslautern, Germany

2 Faculty of Mathematics, Technische Universität Chemnitz, Chemnitz, Germany

3 Department of Applied Mechanics, University of Kaiserslautern, Kaiserslautern, Germany state effective models. We discuss the efficient implementation of our method, and present results of a component scale computation on a benchmark component by using ABAQUS ${ }^{\circledR}$.

Keywords Multiscale simulation - Viscoelasticity . Homogenization - Fiber orientation tensor - FFT based method

\section{Introduction}

\subsection{State of the art}

Composite materials are frequently used in engineering applications. However, the difference in sizes between the reinforcements and the parts generally prohibits a finite element analysis of the component where the underlying mesh resolves the heterogeneities. It is common in engineering practice to overcome this problem by resorting to effective models which characterize the composite's behavior on the component scale.

To identify these models, experimental methods are supported by mean field and computational upscaling techniques (cf. Zaoui [61] and Matouš et al. [35] for respective surveys), mathematically formalized by the theory of homogenization [6].

For nonlinear material behavior or large differences in the elastic properties of the constituents, numerical methods resolving the microstructure on representative volume elements (RVEs) for the mechanical behavior under considerations [28] are often the only accurate option, and a variety of numerical approaches specialized to homogenization problems have been developed $[2,5,40]$. 


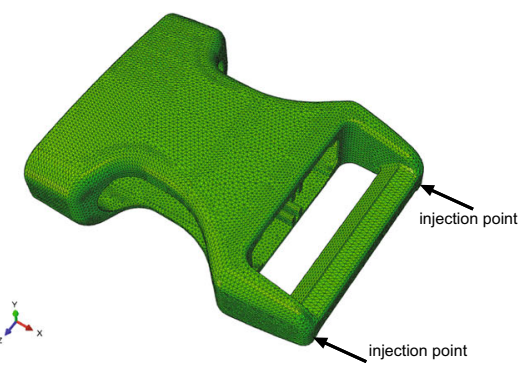

(a)

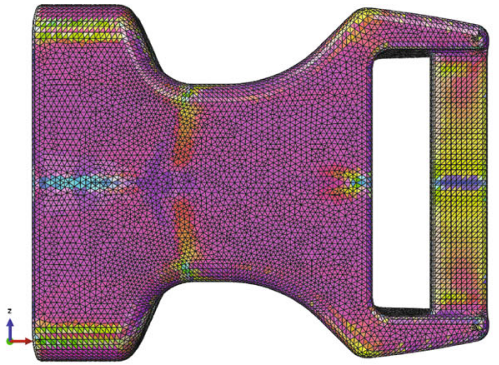

(b)

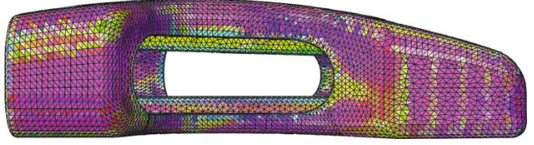

(c)

Fig. 1 Injection molded quick release buckle socket: injection points, finite element mesh, and fiber orientation distribution (see Fig. 3 for the color coding). a Injection points and finite element mesh. b Fiber orientation (bottom view). c Fiber orientation (side view)
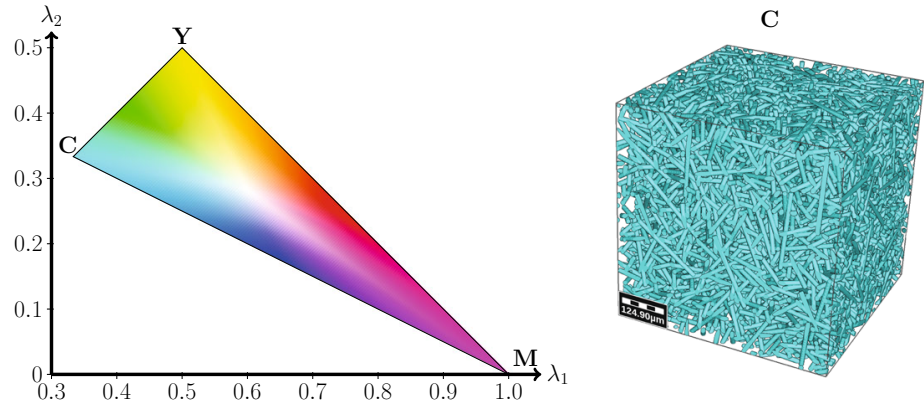
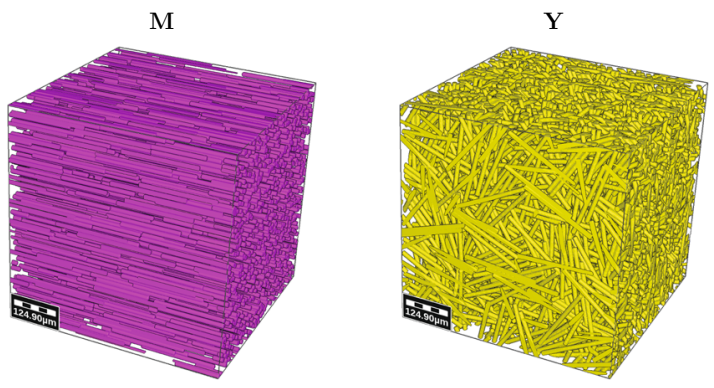

Fig. 2 The fiber orientation reference triangle, showing the two largest principal eigenvalues of the fiber orientation tensor $A$. The extreme orientations are marked in cyan (isotropic), magenta (unidirectional) and yellow (planar), whereas the intermediate orientation states arise from combinations of the colors in CMY format, s.t. the center of mass of the triangle is white. (Color figure online)
Solving two-scale problems with nested volume element problems, known as $\mathrm{FE}^{2}$ analysis or heterogeneous multiscale method [17], is limited by their formidable sizedespite impressive computational results like in MosbyMatouš [38]. The micro-mechanical problems under consideration, however, share the same geometry, but involve different macroscopic loading conditions. Thus, a huge amount of very similar problems is solved. Based on this observation a variety of acceleration techniques have been developed, among them reduced order models $[9,55,59]$, the (non-uniform) transformation field analysis $[16,20,36]$, response surface models $[4,12,46,50,60]$ and machine learning approaches like neural networks $[21,24,33,34,52]$ giving rise to accurate surrogate models with significantly lower computational demands.

Surrogate models can than be used in a database concept [49,51].

\subsection{Problem setting}

Short fiber reinforced composite parts constitute an example for multiscale problems where the parameters determining the microstructure may vary continuously on the macro scale.
For illustration, Fig. 1 shows a quick release buckle socket ${ }^{1}$ for which an injection molding simulation was conducted (see Sect. 4 for the exact simulation parameters). The colors indicate the occurring fiber orientation (FO) see Fig. 2: cyan corresponds to isotropic, yellow to planar and magenta to a unidirectional, i.e. an aligned, fiber orientation state. We see that large parts of the socket exhibit a rather aligned orientation. Yet, there are distinguished parts, like the flat stern where the orientation is mostly planar. Furthermore, there are regions where the melt fronts have collided (welding lines). Capturing the mechanical behavior of these regions is particularly important, as they correspond to weak spots of the structure.

The finite element mesh, cf. Fig. 1a, consists of more than 300,000 elements. This large number of elements is necessary for resolving the component. Notice that the fiber orientation is varying continuously, and large parts of the component are characterized by very similar fiber orientation states. Thus, it appears natural to employ an interpolation ansatz to cover the different orientations. Unfortunately, using representative volume elements (RVEs) for computing the effective mechanical response is, at first sight, incompat-

\footnotetext{
${ }^{1}$ Source for the CAD geometry: https://grabcad.com/library/quickrelease-buckle- $19 \mathrm{~mm}$.
} 
ible with an interpolation scheme: the volume element does not depend continuously on the fiber orientation! The situation is even more delicate: one and the same fiber orientation state might be represented by two completely different volume elements. Thus, the association $\{\mathrm{FO}\} \mapsto\{\mathrm{RVE}\}$ is not even a well-defined function. Similar problems arise if surrogate models of different type, in particular with different internal variables, are used for different orientations. This situation is characteristic for models obtained by model order reduction, for instance.

In this work, we propose a work-around for these problems, based on the insight that the effective stress is a well-defined function of the fiber orientation (and the applied load history).

\subsection{Our contribution and organization of this article}

Within the framework of generalized standard materials $[23,25]$ we propose a method to interpolate arbitrary effective models. Suppose we have a collection of microstructures, indexed by $\{1, \ldots, M\}$. For every microstructure whose constituents are generalized standard materials, the effective mechanical behavior can be described by a generalized standard material, see Suquet [48]. Thus, we obtain a free energy $W_{i}$ and a dissipation potential $\Phi_{i}$ for each index $i$. Notice, however, that $\Phi_{i}$ depends also on an internal variable vector $Q_{i}$, which lives in some space $X_{i}$.

Let the state to be interpolated be described by weights $s_{1}, \ldots, s_{M}$ forming a convex combination, i.e. satisfying

$s_{i} \geq 0, \quad \sum_{i=1}^{M} s_{i}=1$,

the interpolated free energy and dissipation potential read

$W(E, Q)=\sum_{i=1}^{M} s_{i} W_{i}\left(E, Q_{i}\right)$

and

$\Phi(\dot{Q})=\sum_{i=1}^{M} s_{i} \Phi_{i}\left(\dot{Q}_{i}\right)$,

where $E$ denotes the applied macroscopic strain and $Q=$ $\left(Q_{1}, \ldots, Q_{M}\right)$ collects all internal variables. This construction naturally preserves convexity (and continuity) properties of the potentials, and yields the desired evolution equations with effective properties continuously varying between the microstructures. For a typical simplicial triangulation in the $d$-dimensional space describing the microstructure, each microstructure to be interpolated is surrounded by $d+1$ nodes. Consequently, only $d+1$ internal variables need to be tracked instead of the full $M$. This property contrasts with other methods, for instance the method of pseudo-grains $[13,15]$.

In the context of short fiber reinforced composites we apply the proposed method to the interpolation of fiber reinforced structures described by the fiber orientation tensor of second order, see Sect. 2, corresponding-if objectivity is accounted for-to a two-dimensional configuration space.

To study the creeping behavior of short fiber reinforced composites, we equip the matrix with a Burgers model for PA66 [57,58], use FFT-based computational homogenization $[39,40]$ to carry out the microstructure computations and identify a surrogate model by Schapery's collocation method [43], see Sect. 3. Last but not least we check the accuracy of the fiber orientation interpolation and investigate the behavior of the quick release buckle socket of Fig. 1, see Sect. 4. Care has been taken to ensure applicability of the scheme in state of the art engineering computations. For the micromechanical simulation, we use the Fraunhofer ITWM software FeelMath [18], distributed as the ElastoDict module of GeoDict [22]. The database containing the different effective models is filled by a script written in Python [42], and can be accessed by a user-defined material function, which is compatible with the UMAT interface of ABAQUS [1].

\section{Fiber orientation interpolation}

\subsection{The fiber orientation tensor}

Short fiber reinforced composites with fibers of equal length are locally characterized by the direction of their fiber reinforcements. Suppose a given volume $V$ comprises a number of fibers with directions $p_{1}, \ldots, p_{N} \in S^{2}$, where $S^{2}$ denotes the the unit sphere, $N$ can be very large. Then, the AdvaniTucker (second order) fiber orientation tensor $A$ [3] computes via

$A=\frac{1}{N} \sum_{i=1}^{N} p_{i} \otimes p_{i}$

The fiber orientation tensor $A$ is a symmetric positive semidefinite $3 \times 3$ tensor with $\operatorname{tr}(A)=1$ and represents a compact measure of the fiber orientation distribution in the volume $V$. For instance, $A$ is degenerate precisely if all fibers $\left\{p_{i}\right\}_{i=1}^{N}$ are contained in a single great circle, i.e. if the fiber orientation is planar. Furthermore, $A$ is of the form $A=p \otimes p$ for a direction $p$ if and only if all fibers $p_{i}$ point in direction $p$ (or, equivalently, in direction $-p$ ).

$A$ carries a limited amount of information, as it comprises only five independent degrees of freedom. Still, it is the principal quantity of interest for commercial injec- 
tion and compression molding simulations, as it may vary for every Gauss point of the FEM Mesh for processed part and plausible higher order momenta are recovered by closure approximations (see Montgomery-Smith et al. [37] for a recent overview). For extensions to higher moment tensors compare Jack-Smith [29]. A principal component analysis of $A$ reveals further information on the fiber orientation. Spectrally decomposing $A$ leads to the expression

$\Lambda=R A R^{T}, \quad \Lambda=\operatorname{diag}\left(\lambda_{1}, \lambda_{2}, \lambda_{3}\right)$

with an orthogonal matrix $R$ and a diagonal matrix $\Lambda$ with sorted eigenvalues $\lambda_{1} \geq \lambda_{2} \geq \lambda_{3}$. The columns $r_{1}, r_{2}$ and $r_{3}$ of $R$ represent the principal fiber directions, and the eigenvalues $\lambda_{i}$ describe the probability of finding fibers in direction $r_{i}$, as the $\lambda_{i}$ are non-negative and sum to one. In particular, up to an orthogonal transformation, the fiber orientation tensor $A$ can be described by by two positive real numbers $\lambda_{1}$ and $\lambda_{2}$ satisfying the two inequalities

$\frac{1}{3} \leq \lambda_{1} \leq 1 \quad$ and $\quad 1-2 \lambda_{1} \leq \lambda_{2} \leq \lambda_{1}$,

which geometrically corresponds to a planar triangle, cf. Fig. 2.

\subsection{The framework of generalized standard materials}

For the constitutive modelling we rely upon the two-potential framework of the generalized standard materials [23,25]. The constitutive relationships are derived from two thermodynamic potentials, the Helmholtz free energy density $w(\varepsilon, q)$ and the dissipation potential $\phi(\dot{q})$ which are convex functions of the state variables, the infinitesimal strain $\varepsilon$ and other internal variables $q$, and their time-derivative. The free energy $w$ is the energy available in the system to trigger its evolution, whereas $\phi$ describes the evolution of the irreversible mechanisms.

In a first step, the thermodynamic forces stored in the materials are derived via

$\sigma=\frac{\partial w}{\partial \varepsilon}(\varepsilon, q)$ and $\mathcal{Q}=-\frac{\partial w}{\partial q}(\varepsilon, q)$.

Then, complementary laws relate the rate of the state variables and the forces acting upon them, i.e.

$\mathcal{Q}=\frac{\partial \phi}{\partial \dot{q}}(\dot{q})$

Combining these formulae leads to the equations

$\sigma=\frac{\partial w}{\partial \varepsilon}(\varepsilon, q)$ and $\frac{\partial w}{\partial q}(\varepsilon, q)+\frac{\partial \phi}{\partial \dot{q}}(\dot{q})=0$.

\subsection{Effective properties of short fiber reinforced plastics and the invariance principle}

From the work of Suquet $[47,48]$ it is known that the macroscopic response of a microstructure with generalized standard material constituents is described by a generalized standard material, possibly with an infinite number of internal variables. Thus, we assume, that for every fiber orientation tensor $A$ we have an effective free energy $W_{A}$, depending on the macroscopic strain $E$ and a vector of internal variables $Q_{A}$, which, for simplicity of exposition, we assume to be a collection of two-tensors, and an effective dissipation potential $\Phi_{A}$, depending on the rate of change of the internal variables $\dot{Q}_{A}$. Thus, the macroscopic constitutive relations according to (4) read

$\Sigma=\frac{\partial W_{A}}{\partial E}\left(E, Q_{A}\right)$

and

$\frac{\partial W_{A}}{\partial Q_{A}}\left(E, Q_{A}\right)+\frac{\partial \Phi_{A}}{\partial \dot{Q}_{A}}\left(\dot{Q}_{A}\right)=0$,

where $\Sigma$ is the macroscopic stress.

However, the material laws for different fiber orientation states are not independent. Due to objectivity and material frame indifference, both the free energy and the dissipation potential are invariant w.r.t. orthogonal transformations. More precisely, for any fiber orientation $A$, strain $E$, internal variable $Q_{A}$ and orthogonal transformation $R$ we have the equalities

$W_{A}\left(E, Q_{A}\right)=W_{R A R^{T}}\left(R E R^{T}, Q_{R A R^{T}}\right)$

and

$\Phi_{A}\left(\dot{Q}_{A}\right)=\Phi_{R A R^{T}}\left(\dot{Q}_{R A R^{T}}\right)$,

where we used that the fiber orientation is static, $\dot{A}=0$. Differentiating yields the equations

$\Sigma=R^{T}\left[\frac{\partial W_{R A R^{T}}}{\partial E}\left(R E R^{T}, Q_{R A R^{T}}\right)\right] R$

and

$\frac{\partial W_{R A R^{T}}}{\partial Q_{R A R^{T}}}\left(R E R^{T}, Q_{R A R^{T}}\right)+\frac{\partial \Phi_{R A R^{T}}}{\partial \dot{Q}_{R A R^{T}}}\left(\dot{Q}_{R A R^{T}}\right)=0$,

which are equivalent to (5) and (6). The latter observations are illustrated on a unidirectional structure in Fig. 4. To compute the effective stress of the unidirectional structure on the 
left, which can be directed arbitrarily, we can use a unidirectional structure with a fixed direction (on the right), provided we transform the applied strain as well as the internal variables (not shown) accordingly, and do not forget to transform the effective stress back to the reference orientation.

Thus, to derive effective models for all fiber orientation states $A$ it is sufficient to consider the fiber orientations $\Lambda=$ $\operatorname{diag}\left(\lambda_{1}, \lambda_{2}, 1-\lambda_{1}-\lambda_{2}\right)$ with $\lambda_{1}, \lambda_{2}$ lying in the triangle (3). Effective constitutive laws for general orientation tensors are obtained by subsequent orthogonal transformations.

More precisely, suppose the family $\left\{W_{\Lambda}, \Phi_{\Lambda}\right\}_{\Lambda}$ is given, and let $A$ be arbitrary. Spectrally decomposing $A=R^{T} \Lambda R$ leads, for macroscopic strain $E$, to the constitutive law

$$
\begin{aligned}
& \Sigma=R^{T}\left[\frac{\partial W_{\Lambda}}{\partial E}\left(R E R^{T}, Q_{\Lambda}\right)\right] R, \\
& \frac{\partial W_{\Lambda}}{\partial Q_{\Lambda}}\left(R E R^{T}, Q_{\Lambda}\right)+\frac{\partial \Phi_{\Lambda}}{\partial \dot{Q}_{\Lambda}}\left(\dot{Q}_{\Lambda}\right)=0 .
\end{aligned}
$$

The free energy, the dissipation potential and the internal variables live in the diagonalized configuration, whereas both stresses and strains share the frame of the fiber orientation tensor $A$.

\subsection{Fiber orientation interpolation}

In the preceding section we have reduced the problem of finding the effective response of a fiber reinforced composite from a five-dimensional problem, corresponding to the full fiber orientation tensor $A$, to a two-dimensional problem, taking into account the fiber orientation triangle. Still, this triangle consists of an infinite number of points. From physical intuition it is clear that for fixed applied strain the free energy in equilibrium is continuous as a function of the orientation. Thus, if we discretize the fiber orientation triangle of Fig. 2 by a sufficient fine mesh, and associate a constitutive law $\left(W_{\Lambda}, \Phi_{\Lambda}\right)$ to each nodal point $\Lambda$ of the mesh, a subsequent interpolation should capture all effective materials law with sufficient accuracy, provided the triangulation is fine enough, see Fig. 3.

However, care has to be taken as to which quantities can be interpolated. In general, in contrast to macroscopic strains and stresses, it does not make sense to interpolate internal variables, as they live on the current configuration (and fiber orientation state). Thus, it is not clear how to transfer them between different states. Even more striking is the fact that upon discretization the number of internal variables becomes finite, and might differ for distinct fiber orientations. A solution to this dilemma is the following. Suppose $\Lambda$ is a general fiber orientation state in the fiber orientation triangle. For a given triangulation let $\Lambda_{1}, \Lambda_{2}$ and $\Lambda_{3}$ be the nodes of the triangle containing $\Lambda$, with constitutive laws $\left\{W_{\Lambda_{i}}, \Phi_{\Lambda_{i}}\right\}_{i=1,2,3}$. For a non-degenerated triangle there is a

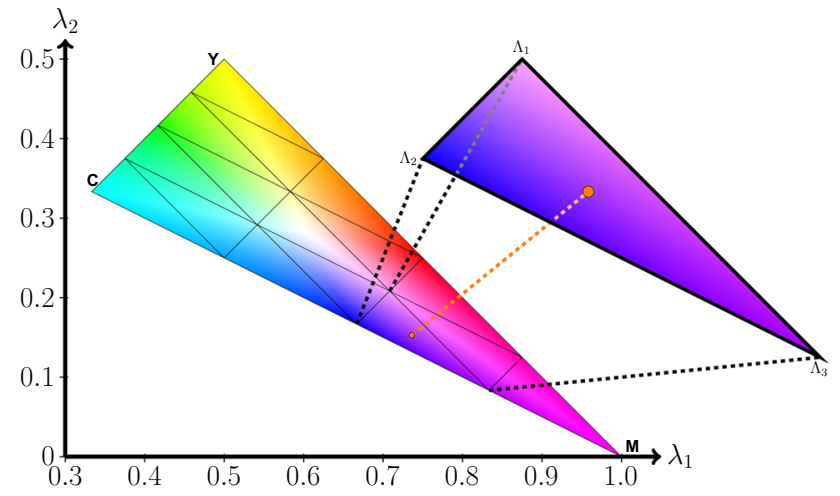

Fig. 3 A triangulation of the fiber orientation triangle. For a given orientation state (orange), the containing triangle is augmented, with nodes $\Lambda_{1}, \Lambda_{2}$ and $\Lambda_{3}$. The coloring encodes the orientation. (Color figure online)

representation as convex linear combination

$\Lambda=s_{1} \Lambda_{1}+s_{2} \Lambda_{2}+s_{3} \Lambda_{3}$

with a unique real triple $\left(s_{1}, s_{2}, s_{3}\right)$ satisfying

$s_{i} \geq 0, \quad(i=1,2,3) \quad$ and $s_{1}+s_{2}+s_{3}=1$.

We associate to $\Lambda$ the free energy

$\widehat{W}_{\Lambda}\left(E, Q_{\Lambda_{1}}, Q_{\Lambda_{2}}, Q_{\Lambda_{3}}\right)=\sum_{i=1}^{3} s_{i} W_{\Lambda_{i}}\left(E, Q_{\Lambda_{i}}\right)$

and the corresponding dissipation potential

$\widehat{\Phi}_{\Lambda}\left(\dot{Q}_{\Lambda_{1}}, \dot{Q}_{\Lambda_{2}}, \dot{Q}_{\Lambda_{3}}\right)=\sum_{i=1}^{3} s_{i} \Phi_{\Lambda_{i}}\left(\dot{Q}_{\Lambda_{i}}\right)$

These two potentials include three sets of internal variables, and are continuous in $\Lambda$ through the $\left(s_{1}, s_{2}, s_{3}\right)$ coefficients (12). Our construction preserves the convexity of both the free energies and the dissipation potentials.

Differentiating (14) according to (4) yields the constitutive law

$$
\begin{aligned}
& \Sigma=\sum_{i=1}^{3} s_{i} \frac{\partial W_{\Lambda_{i}}}{\partial E}\left(E, Q_{\Lambda_{i}}\right), \\
& \frac{\partial W_{\Lambda_{i}}}{\partial Q_{\Lambda_{i}}}\left(E, Q_{\Lambda_{i}}\right)+\frac{\partial \Phi_{\Lambda_{i}}}{\partial \dot{Q}_{\Lambda_{i}}}\left(\dot{Q}_{\Lambda_{i}}\right)=0, \quad i=1,2,3 .
\end{aligned}
$$

Thus, the nodal stresses are interpolated, and the nodal internal variables evolve independently of each other. Of course, if $s_{i}=0$ for some $i=1,2,3$, the evolution for $Q_{\Lambda_{i}}$ becomes irrelevant for the evaluation of the stress. In particular, if $\Lambda=\Lambda_{i}$ for some $i=1,2,3$, we consistently recover the constitutive equations for $\Lambda_{i}$. 
Fig. 4 Illustration of the invariance principle: rotating both the fibers and the loading leads to a rotated stress
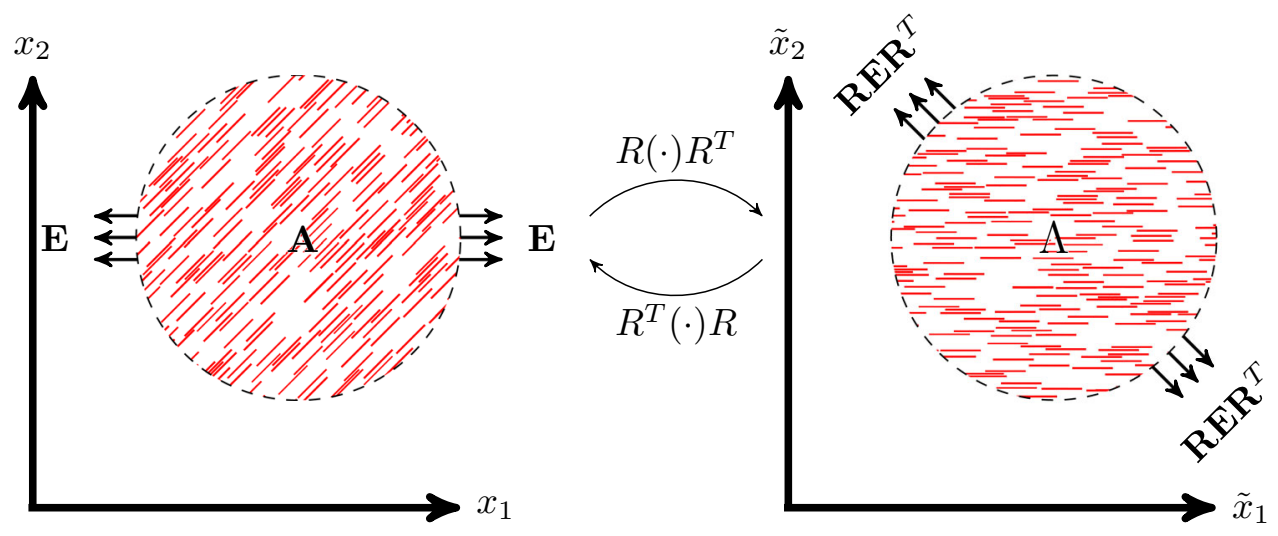

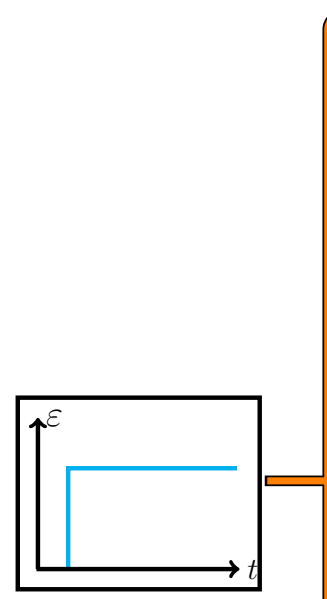

loading
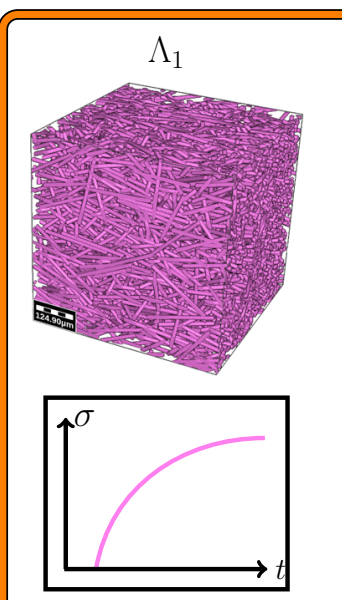

linear interpolation using fiber orientation database
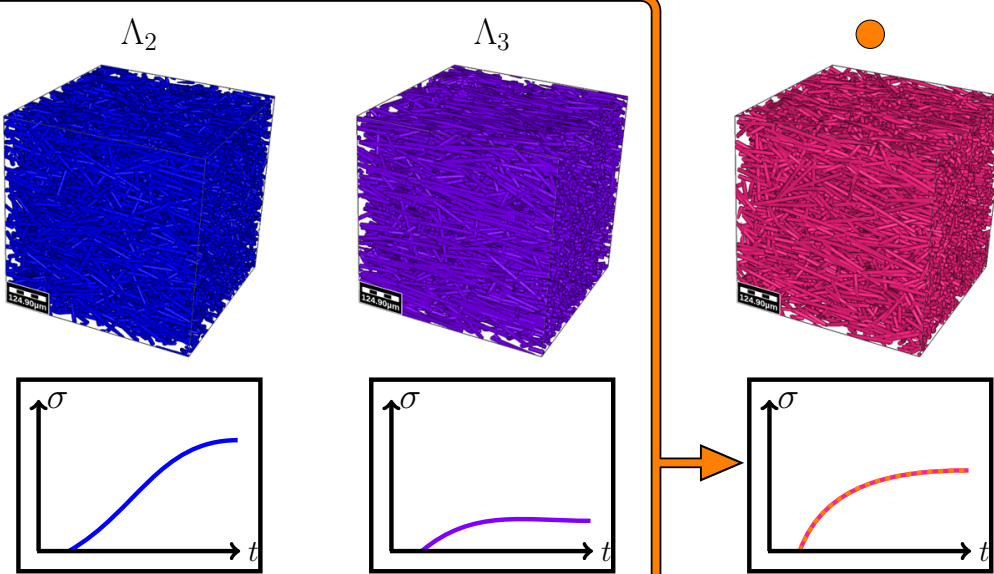

stress response
Fig. 5 Conceptional illustration of the fiber orientation interpolation method for the orientations of Fig. 3. The applied loading gives rise to three stress responses corresponding to the nodes of the triangle. The

In this formulation, three sets of internal variables are stored. Each node receives the same strain, but uses its own constitutive law to produce a stress, and the evolution of the internal variables depends on the node only. The three stresses are averaged according to the convex representation (12), which is meaningful, see Fig. 5.

We have presented the method for a single triangle of the triangulation only. However, the method can be interpreted for the whole triangulation in a similar fashion, where only the adjacent nodal constitutive laws are active. Notice at this point that for every orientation only three material laws are required, regardless of the number of nodes used for the triangulation of the fiber orientation triangle. This property contrasts starkly with the method of pseudo-grains $[13,15]$, which requires the full number of grains to be evaluated for calculating the effective response of the composite.

The fiber orientation interpolation method can be summarized as follows. stress responses are averaged according to the convex coordinates of the orange orientation, giving rise to the macroscopic stress. (Color figure online)

1. Offline phase: triangulate the fiber orientation triangle, cf. Fig. 3, and compute an effective model for each node in the triangulation

2. Online phase: for each macroscopic Gauss point and given strain $E$

- Spectrally decompose the local fiber orientation tensor $A=R^{T} \Lambda R$, cf. (2)

- Determine the local triangle $T$ such that $\Lambda \in$ $T=\operatorname{conv}\left\{\Lambda_{1}, \Lambda_{2}, \Lambda_{3}\right\}$ with nodes $\Lambda_{1}, \Lambda_{2}, \Lambda_{3}$ and weights $s_{1}, s_{2}, s_{3}$, cf. (12)

- Transform the strain $E \mapsto R E R^{T}$, compute the local stress $\Sigma_{i}$ and update the internal variable $Q_{\Lambda_{i}}$ at node $\Lambda_{i}$

- Return the effective stress $\Sigma=R^{T}\left[\sum_{i=1}^{3} s_{i} \Sigma_{i}\right] R$

- Return the effective consistent tangent matrix $\mathbb{C}=$ $\mathbb{R}^{-1}:\left[\sum_{i=1}^{3} s_{i} \mathbb{C}_{i}\right]: \mathbb{R}$, where $\mathbb{C}_{i}$ is the consistent tangent at $\Sigma_{i}$ in the transformed orientation, $\mathbb{R}$ is 
the action of $R$ on two-tensors $\mathbb{R}_{i j k l}=R_{i k} R_{j l}$, and $\mathbb{R}_{i j k l}^{-1}=R_{k i} R_{l j}$

In this work, the offline phase is implemented in Python [42]: for a given triangulation of the fiber orientation triangle, the micromechanical solver (see Sect. 3.3) is called for each node of the triangulation and its results are used to identify the parameters of a surrogate model. As output, for each element in the fiber orientation triangulation, an ordered set of nodes with corresponding ABAQUS [1] UMATs and identified parameters for the UMAT are stored in an XML file. Once the component to be simulated is chosen, for each element of the component's finite element mesh, the eigendecomposition of the fiber orientation is computed, the Euler angles of the rotation matrix, the weights associated to the interpolation (12) and the corresponding element of the fiber orientation triangle are determined and stored.

For the online ABAQUS computation a dummy UMAT is used, which takes Euler angles, weights and the fiber orientation element as input parameters, and calls the three UMATs associated to the adjacent nodes of the fiber orientation triangle.

\section{Identifying a viscoelastic surrogate model using FFT-based computational homogenization and Schapery's collocation method}

To test the orientation interpolation technique we consider a fiber reinforced polymer with linearly viscoelastic matrix and linear elastic reinforcements. Linear viscoelasticity has the advantage that on the one hand the derivation of effective models is comparatively well-understood, but on the other hand due to the dependence on the material history complex constitutive behavior can be observed.

\subsection{Generating fiber-filled volume elements}

To generate periodic volume elements with prescribed fiber orientation and volume fraction we rely upon the Sequential Addition and Migration (SAM) method [44]. As input parameters, the fiber volume fraction $\phi$, the second order fiber orientation tensor $A$, the fiber length $L$, the fiber diameter $D$ and the edge length $L_{x}\left(=L_{y}=L_{z}\right)$ of the cubical volume element needs to be specified. In a preliminary step, the required number $N$ of fibers is calculated, s.t. the fiber volume fraction $\phi$ is matched with highest precision. Furthermore, the fourth order fiber orientation tensor $\mathbb{A}$ is computed from the $A$ tensor with the help of the exact closure approximation [37].

In the first step of SAM, $N$ overlapping cylindrical fibers are randomly placed in the volume. Then, in the second step, the fibers are displaced and rotated, s.t. the overlap is removed
Fig. 6 Rheological diagram of Burgers' model

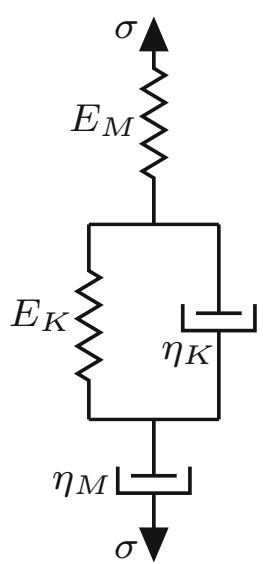

and the prescribed fiber orientation tensor $\mathbb{A}$ is matched to prescribed accuracy (typically $10^{-5}$ absolute error in $L^{2}$ of the Voigt matrices).

The SAM algorithm is unable to generate purely planar fiber orientation states, i.e. (up to orthogonal transformation) states corresponding to a fiber orientation tensor of the form

$A=\operatorname{diag}\left(\lambda_{1}, \lambda_{2}, 0\right)$.

However, these states do not appear in practice for industrial fiber volume fractions. Indeed, suppose a collection of fibers with directions $p_{1}, \ldots, p_{N}$ is given. This system is purely planar in the $x-y$-plane precisely if $p_{i} \cdot e_{z}=0$ for all $i=1, \ldots, N$, i.e. all fibers have vanishing $z$-component. In particular, no scatter is allowed in the $z$-direction.

For the study at hand, we force $\lambda_{3}$ to be at least 0.01 . These orientation tensors are sufficiently close to planar for practical studies, and can easily be generated by the SAM method.

\subsection{The Burgers model for the viscoelastic behavior of the matrix}

As matrix material we consider a commercial polyamide 6.6 (PA66, DuPont Zytel 101), for which experimental creep curves and fitted viscoelastic model parameters are available in the literature, see Yang et al. [57,58].

The Burgers model, a classic in the literature [53], is widely used for the linear viscoelastic behavior of ideal thermoplastics. The rheological diagram is shown in Fig. 6 with a Maxwell and a Kelvin unit connected in series. The Burgers model exhibits a stationary creep rate after relaxation of the Kelvin element, includes an irreversible part of the viscous strain after unloading and the stress in a relaxation test approaches 0 as $t \nearrow \infty$.

According to Burgers' model, for an applied tensile stress of $\sigma_{0}$, the tensile strain computes as

$$
\varepsilon(t)=\sigma_{0}\left[\frac{1}{E_{M}}+\frac{1}{E_{K}}\left(1-e^{-t / \tau}\right)+\frac{t}{\eta_{M}}\right],
$$


where $E_{M}$ and $\eta_{M}$ are the Young's modulus and viscosity of the Maxwell spring and dashpot, respectively, and $E_{K}$ and $\eta_{K}$ are the Young's modulus and viscosity of the Kelvin spring and dashpot, respectively, whereas $\tau=\eta_{K} / E_{K}$ represents the retardation time.

Reciprocally, for an applied tensile strain of $\varepsilon_{0}$, the tensile stress attains the form [19]

$\sigma(t)=\frac{\varepsilon_{0}}{A}\left[\left(q_{1}-q_{2} r_{1}\right) e^{-r_{1} t}-\left(q_{1}-q_{2} r_{2}\right) e^{-r_{2} t}\right]$

with

$p_{1}=\frac{\eta_{M}}{E_{M}}+\frac{\eta_{M}}{E_{K}}+\tau, \quad p_{2}=\frac{\eta_{M}}{E_{M} \tau}$,

$q_{1}=\eta_{M}, \quad q_{2}=\eta_{M} \tau, \quad r_{1 / 2}=\frac{p_{1} \mp A}{2 p_{2}}$,

$A=\sqrt{p_{1}^{2}-4 p_{2}}$.

Using the latter representation it is not difficult to exhibit Burgers' model as a generalized standard material. However, for practical purposes the creep function representation (16) is advantageous. Following Lai-Bakker [32] and Woldekidan [56] we obtain a 3D model by assuming Poisson's ratio $v=0.38$ to be constant over the complete time history. This is a common assumption for glassy or semi-crystalline thermoplastic polymers as the investigated matrix material. We arrive at the hereditary integral formulation

$\varepsilon(t)=\int_{0}^{t} \mathbb{J}(t-s): \dot{\sigma}(s) d s$,

relating the stress rate $\dot{\sigma}$ to the strain $\varepsilon$ using the viscoelastic intensification tensor

$\mathrm{J}(t)=\mathbb{D}_{0}+t \mathbb{F}+\mathbb{D}_{1}\left(1-e^{-t / \tau}\right)$

for $t \geq 0$ and zero otherwise. Here, $\mathbb{F}$ denotes the creep compliance and $\mathbb{D}_{0}$ is the instantaneous compliance. If $\mathbb{F}=0$, then $\mathbb{D}_{0}+\mathbb{D}_{1}$ corresponds to the relaxed (elastic) compliance. In formulae, the action of the three compliances on a test stress $S$ read

$$
\begin{aligned}
\mathbb{D}_{0}: S & =\frac{1+v}{E_{M}} S-\frac{v}{E_{M}} \operatorname{tr}(S) \mathrm{Id}, \\
\mathbb{D}_{1}: S & =\frac{1+v}{E_{K}} S-\frac{v}{E_{K}} \operatorname{tr}(S) \mathrm{Id}, \\
\mathbb{F}: S & =\frac{1+v}{\eta_{M}} S-\frac{v}{\eta_{M}} \operatorname{tr}(S) \mathrm{Id},
\end{aligned}
$$

where Id is the $3 \times 3$ identity matrix.

The chosen material parameters are summarized in Table 1 , corresponding to the $\sigma_{0}=20 \mathrm{MPa}$ and $T=23^{\circ} \mathrm{C}$ fitted parameters of Yang et al. [58].
Table 1 Viscoelastic matrix material parameters [58] for Burgers' model

\begin{tabular}{llll}
\hline$E_{M}[\mathrm{MPa}]$ & $E_{K}[\mathrm{MPa}]$ & $\eta_{M}[\mathrm{GPah}]$ & $\tau[\mathrm{h}]$ \\
\hline 3709 & 16.617 & 889 & 14.2 \\
\hline
\end{tabular}

\subsection{Computational homogenization of linear viscoelasticity}

Following Hashin [26,27] suppose $Y \subseteq \mathbb{R}^{3}$ is a cuboid (for periodic boundary conditions) and let a heterogeneous linear viscoelastic medium be given in terms of the local relaxation function

$\mathrm{J}: Y \times[0, \infty) \longrightarrow \mathbb{R}^{3} \otimes \mathbb{R}^{3} \otimes \mathbb{R}^{3} \otimes \mathbb{R}^{3}$,

s.t. for every $(x, t) \in Y \times[0, \infty), \mathrm{J}(x, t)$ has minor as well as major symmetries and is coercive. To homogenize, i.e. to compute effective linear viscoelastic properties, for given macroscopic stress history $\Sigma:[0, \infty) \rightarrow \operatorname{Sym}(3)$, where $\operatorname{Sym}(3)$ denotes the linear space of symmetric $3 \times 3$ matrices, we seek the local stress field $\sigma: Y \times[0, \infty) \rightarrow$ $\operatorname{Sym}(3)$, the periodic local displacement fluctuation field $u: Y \times[0, \infty) \rightarrow \mathbb{R}^{3}$ and the macroscopic strain history $E:[0, \infty) \rightarrow \operatorname{Sym}(3)$, s.t. the following equations are satisfied for every $t \in[0, \infty)$ :

\section{- balance of linear momentum}

$\operatorname{div} \sigma(t)=0$,

- compatibility

$$
\varepsilon(x, t)=E(t)+\nabla^{s} u(x, t) \text { for every } x \in Y,
$$

- linear viscoelastic material law in relaxation function form

$$
\varepsilon(x, t)=\int_{0}^{t} \mathrm{~J}(x, t-s): \dot{\sigma}(x, s) d s \quad \text { for every } x \in Y,
$$

- fixed macroscopic stress

$$
\langle\sigma(t)\rangle_{Y}=\Sigma(t)
$$

Here, $\varepsilon$ is the local strain field, we write $\sigma(t) \equiv \sigma(\cdot, t)$ for the sake of notational clarity, $\nabla^{s}$ denotes the symmetrized gradient, and $\langle\cdot\rangle_{Y}$ stands for the averaging operator over Y. Without loss of generality, $\langle u(t)\rangle_{Y}=0$ can be enforced for every $t \geq 0$.

The quantity of principal interest is the macroscopic strain field history $E$. Due to the linearity and the time-invariance 
of the mapping $\Sigma \mapsto E$, there is a homogenized relaxation function $\mathbb{J}^{\text {hom }}:[0, \infty) \rightarrow \mathbb{R}^{3} \otimes \mathbb{R}^{3} \otimes \mathbb{R}^{3} \otimes \mathbb{R}^{3}$ with both minor and major symmetries, s.t. we can write

$E(t)=\int_{0}^{t} \mathrm{~J}^{h o m}(t-s): \dot{\Sigma}(s) d s$.

Indeed, $\mathrm{J}^{\text {hom }}$ arises by choosing

$\Sigma(t)= \begin{cases}0, & t<0 \\ \Sigma_{0}, & t \geq 0\end{cases}$

for six linearly independent $\Sigma_{0} \in \operatorname{Sym}(d)$, as the corresponding macroscopic strain fields satisfy

$E(t)=J^{h o m}(t): \Sigma_{0}$

Thus, the effective creeping behavior completely determines the full linear viscoelastic effective behavior.

We solve (18)-(21) in the standard way [30] by resorting to a time discretization $0=t_{0}<t_{1}<t_{2}<\cdots$, s.t., for every time step, (18)-(21) becomes a linear elastic problem with eigenstrain, i.e. we seek, suppressing time indices, a strain $E \in \operatorname{Sym}(d)$ and a periodic displacement fluctuation $u: Y \rightarrow \mathbb{R}^{3}$ with zero mean, s.t. the equations

$$
\begin{aligned}
\operatorname{div} \sigma & =0, \\
E+\nabla^{s} u & =\mathbb{D}^{\tan }: \sigma+\alpha, \\
\langle\sigma\rangle_{Y} & =\Sigma,
\end{aligned}
$$

are satisfied, where $\mathbb{D}^{\text {tan }}$ is a tangential compliance tensor and $\alpha$ stands for an eigenstrain depending on quantities computed at the previous time step. (23) constitutes a standard linear elastic homogenization problem with eigenstress and stress "boundary conditions", for which a variety of solution techniques are available.

FFT-based computational homogenization $[39,40]$ constitutes our method of choice. For this method, a discretization on a regular grid or mesh decomposing $Y$ is chosen. Due to the regularity of the grid and the periodic boundary conditions, problems of the form (23) with homogeneous $\mathbb{D}^{\tan }$ can be solved directly in terms of the discrete Fourier transform. The Operator of this auxiliary homogeneous problem serves to precondition the linear system (23).

For the problem at hand, we choose a discretization on a staggered grid [45], the stress-based formulation of Bhattacharya-Suquet [11] and the conjugate gradient method [62] for the resolution of (23). All mentioned methods are integrated into the Fraunhofer ITWM C++ code FeelMath [18].

\subsection{On the resolution and the RVE size necessary for the precomputations}

In this section we investigate the necessary resolution and representative volume element (RVE) size for solving (18)(21), or, equivalently, (23), to engineering accuracy.

We consider short $E$-glass fiber reinforcements with elastic parameters $E=72 \mathrm{GPa}$ and $v=0.22$, length of $200 \mu \mathrm{m}$, aspect ratio $r_{a}=20$ and fiber volume fraction $\phi \approx 17 \%$, which corresponds to a fiber mass fraction of $30 \%$ in the PA66 matrix with the mechanical properties described in Sect. 3.2. Recall from (17) that for creeping with stress $\sigma_{0}$, Burgers' model predicts a strain

$\varepsilon(t)=\mathbb{D}_{0}: \sigma_{0}+t \mathbb{F}: \sigma_{0}+\left(1-e^{t / \tau}\right) \mathbb{D}_{1}: \sigma_{0}$

in the matrix, whereas the glass fibers behave linear elastic (in particular, time independent)

$\varepsilon=\mathbb{D}^{\text {glass }}: \sigma_{0}$.

To investigate the viscoelastic behavior, we consider two extreme cases:

Case 1 The instantaneous response, corresponding to $t=$ 0 , i.e. (24) becomes simply the linear elastic relationship $\varepsilon=\mathbb{D}_{0}: \sigma_{0}$

Case 2 The creep rate at infinity. Differentiating (24) in time yields

$$
\dot{\varepsilon}(t)=\mathbb{F}: \sigma_{0}+\tau^{-1} e^{-t / \tau} \mathbb{D}_{1}: \sigma_{0},
$$

i.e., $\lim _{t \rightarrow \infty} \dot{\varepsilon}(t)=\mathbb{F}: \sigma_{0}$, whereas $\dot{\varepsilon}=0$ in the fibers. To study the creep rate at infinity, we study the linear elastic problem with local compliance

$$
\mathbb{D}(x)= \begin{cases}\mathbb{F}, & x \text { in the matrix } \\ 0, & \text { in the fiber. }\end{cases}
$$

As the fibers are rigid, this problem is much more difficult to solve than the instantaneous elastic problem.

As a first test we study the resolution necessary to obtain accurate effective properties, both for the instantaneous elastic case and the creep rate at infinity. For this purpose, we consider cubical elements with an edge length of $L_{x}=$ $L_{y}=L_{z}=600 \mu \mathrm{m}$, corresponding to thrice the fiber length $L=200 \mu \mathrm{m}$. We choose the extreme orientations, i.e. unidirectional (ud), planar isotropic and (3D) isotropic.

We have chosen to check four different resolutions, measured in voxels per fiber diameter. Recall that the fibers have a diameter of $10 \mu \mathrm{m}$, so that 5 voxels per diameter correspond to a voxel edge length of $2 \mu \mathrm{m}$, i.e. the $(600 \mu \mathrm{m})^{3}$ volume is discretized by $300^{3}$ elements. Accordingly, 10, 15 and 20 


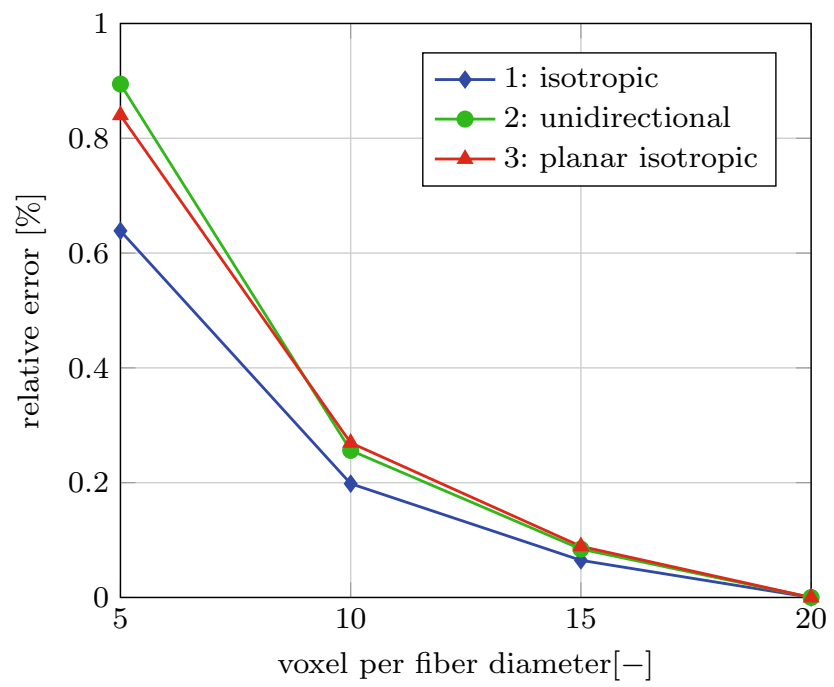

(a)

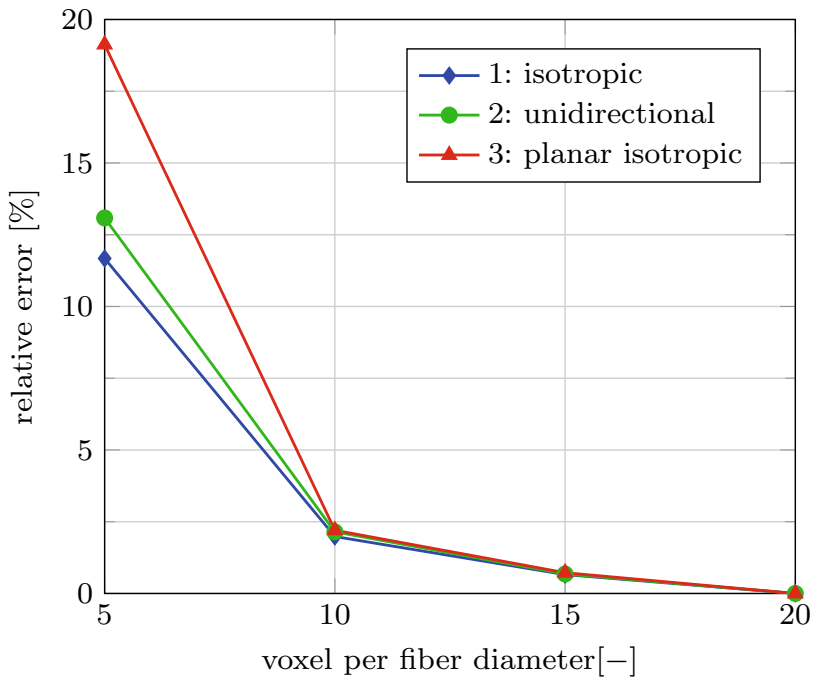

(b)

Fig. 7 Relative error of the stiffness $\mathbb{C}^{h o m}$ and viscosity $\mathbb{V}^{h o m}$ for different resolutions. a Relative error of the stiffness $\mathbb{C}^{\text {hom }}$. b Relative error of the viscosity $\mathbb{V}^{h o m}$

voxels per diameter correspond to $600^{3}, 900^{3}$ and $1200^{3}$ elements, respectively.

For each of these resolutions, the homogenized instantaneous elastic tensor $\mathbb{C}^{h o m}$ was computed (Case 1). The relative error in the Frobenius norm of the corresponding Voigt matrices, measured relative to the highest resolution $1200^{3}$, is shown in Fig. 7a. Even for the crudest resolution, the relative error for all three considered orientations was below $1 \%$.

The same computation was carried out for the long-term, i.e. viscous, response (Case 2). Upon replacing the strain rate $\dot{\varepsilon}$ by the strain $\varepsilon$ in (26) the problem is formally equivalent to an elasticity problem with rigid fibers and elastic matrix. The computed homogenized elastic tensor for the equivalent elastic problem is in fact a homogenized viscous tensor $\mathbb{V}^{\text {hom }}$, and the relative errors of the results are plotted in Fig. 7b, again relative to the highest resolution.

For the crudest resolution, the relative errors exceed 10\% for all three considered orientations. The highest error of about $19.1 \%$ occurs for the planar fiber orientation state, and the lowest error $(11.7 \%)$ is reached for the isotropic orientation. Doubling the resolution decreases the error for all three orientations below $2.5 \%$. For $900^{3}$, the relative error is even smaller than $1 \%$.

We see that in contrast to the instantaneous elastic case computing the long term viscous response requires a comparatively high resolution of 10 voxels per fiber diameter, i.e. a resolution of $1 \mu \mathrm{m}$. We fix the latter resolution for the succeeding. Next we determine the size of a volume element to be representative (see Bella and Otto [7] for recent mathematical findings in the context of linear elasticity) for the homogenized viscoelastic response, see Sect. 3.3, measured in terms of the fiber length $L=200 \mu \mathrm{m}$. With the resolution of $1 \mu \mathrm{m}$, the investigated volume elements comprise $400^{3}$, $600^{3}, 800^{3}, 1000^{3}$ and $1200^{3}$ voxels. To get an impression about the relative size of the volumes, compare Fig. 8, where volume elements with isotropic fiber orientation are shown. The number of fibers corresponding to each volume element size is listed in Table 2.

To study the size of an RVE, we compute the effective elastic/viscous tensors for the different sample sizes and compare these to the result obtained for the largest sample $L_{x}=6 \times L$. It should be mentioned that the variance of different realizations for a fixed unit cell length is negligible, as both the fiber volume fraction and the fourth order fiber orientation tensor are fixed with high accuracy by the SAM method, cf. Schneider [44]. Hence, only the systematic error is measured. For the instantaneous elastic case, see Fig. 9a, the relative error for all resolutions and orientations considered is well below $0.5 \%$, and can be considered negligible. In particular, for the elastic response, twice the fiber length is a sufficient edge length for a representative volume element.

In the viscous case, see Fig. 9b, the relative errors are about an order of magnitude larger than for the elastic case. The unidirectional fiber orientation leads to the largest errors, whereas the isotropic state induces the lowest errors, with the planar case in between these extremes. For the smallest edge length, the relative error is still comparatively large, with about $4 \%$ for the unidirectional fiber orientation. We consider an edge length of thrice the fiber length as sufficient, since both the planar isotropic and isotropic fiber orientation states lead to a relative error of about $1 \%$, and the unidirectional case is captured with $<2.5 \%$ relative error. Table 3 lists the number of iterations necessary for resolving the unit 


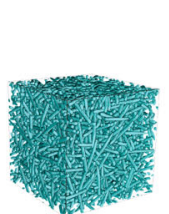

(a)

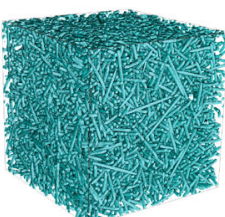

(b)

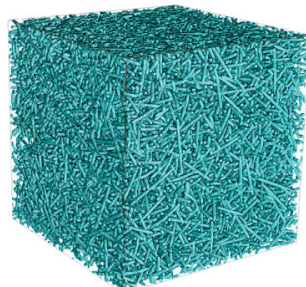

(c)

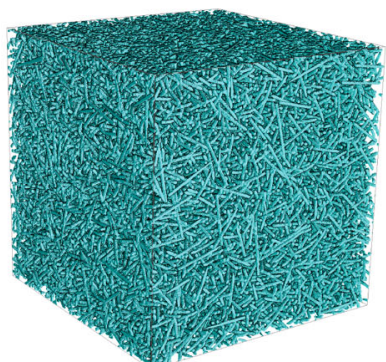

(d)

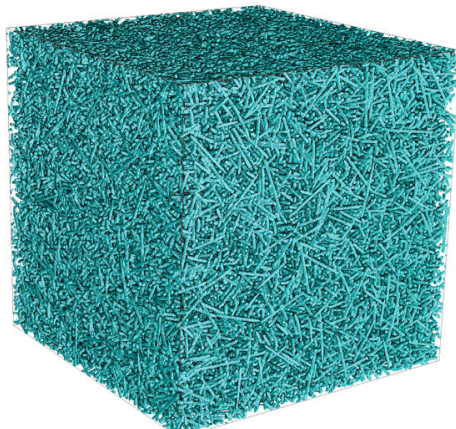

(e)

$L_{x}=2 \times L \quad L_{x}=3 \times L$

$L_{x}=5 \times L$

$L_{x}=6 \times L$

Fig. 8 Volume elements with different edge lengths inside a maximal volume and isotropic fiber orientation tensor. a $L_{x}=2 \times L$. b $L_{x}=3 \times L$. c $L_{x}=4 \times L$. d $L_{x}=5 \times L$. e $L_{x}=6 \times L$

Table 2 Number of fibers for different volume element sizes

\begin{tabular}{llllll}
\hline Edge length & $2 \times L$ & $3 \times L$ & $4 \times L$ & $5 \times L$ & $6 \times L$ \\
\hline Number of fibers & 686 & 2298 & 5447 & 10,640 & 18,386 \\
\hline
\end{tabular}

cell problems for different volume element sizes, both for the elastic and the purely viscous response, measured as the average of the six load cases. The dual formulation of Bhattacharya-Suquet [10] was solved by the conjugate gradient method up to a relative residual of $10^{-5}$. The iteration count mainly depends on the material contrast, is almost independent of the volume element size, but depends slightly on the orientation. For the unidirectional state, a lower number of iterations suffices compared to the planar and 3D isotropic

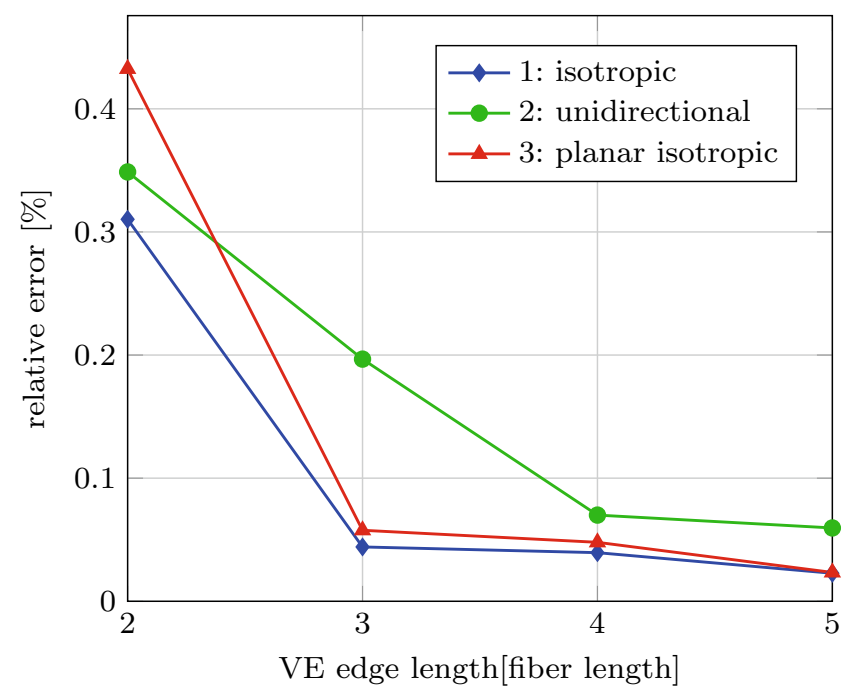

(a) structures. The viscous computations increase the iteration count almost by a factor of 20 compared to the elastic computations.

\subsection{Schapery's collocation method}

To obtain a surrogate model for the homogenized linear viscoelastic behavior of a heterogeneous Burgers medium we rely upon Schapery's collocation method [43]. Recall that the heterogeneous linear viscoelastic material law reads, for every microscopic point $x \in Y$,

$\varepsilon(x, t)=\int_{0}^{t} \mathrm{~J}(x, t-\tau): \dot{\sigma}(x, \tau) d \tau$

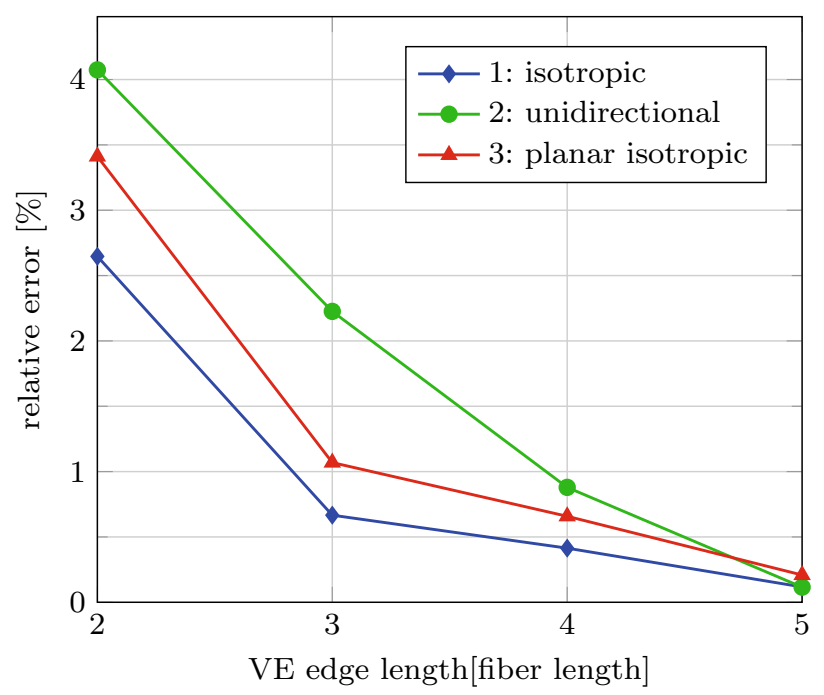

(b)

Fig. 9 Relative error of the stiffness $\mathbb{C}^{h o m}$ and viscosity $\mathbb{V}^{\text {hom }}$ for different volume element sizes. a Relative error of the stiffness $\mathbb{C}^{\text {hom }}$. b Relative error of the viscosity $\mathbb{V}^{\text {hom }}$ 
Table 3 Average number of iterations per load case to compute effective tensors for different volume element sizes

\begin{tabular}{lllll}
\hline & \multicolumn{4}{l}{ Volume element size } \\
\cline { 2 - 5 } Orientation & $2 \times L$ & $3 \times L$ & $4 \times L$ & $5 \times L$ \\
\hline (a) Stiffness & & & & \\
Isotropic & 25.0 & 22.2 & 23.5 & 24.2 \\
Unidirectional & 19.5 & 19.5 & 19.5 & 19.5 \\
Planar isotropic & 19.7 & 19.8 & 21.2 & 20.8 \\
(b) Viscosity & & & & \\
Isotropic & 498.3 & 495.2 & 492.2 & 490.0 \\
Unidirectional & 461.3 & 454.8 & 451.2 & 450.7 \\
Planar isotropic & 494.8 & 486.3 & 486.5 & 485.0 \\
\hline
\end{tabular}

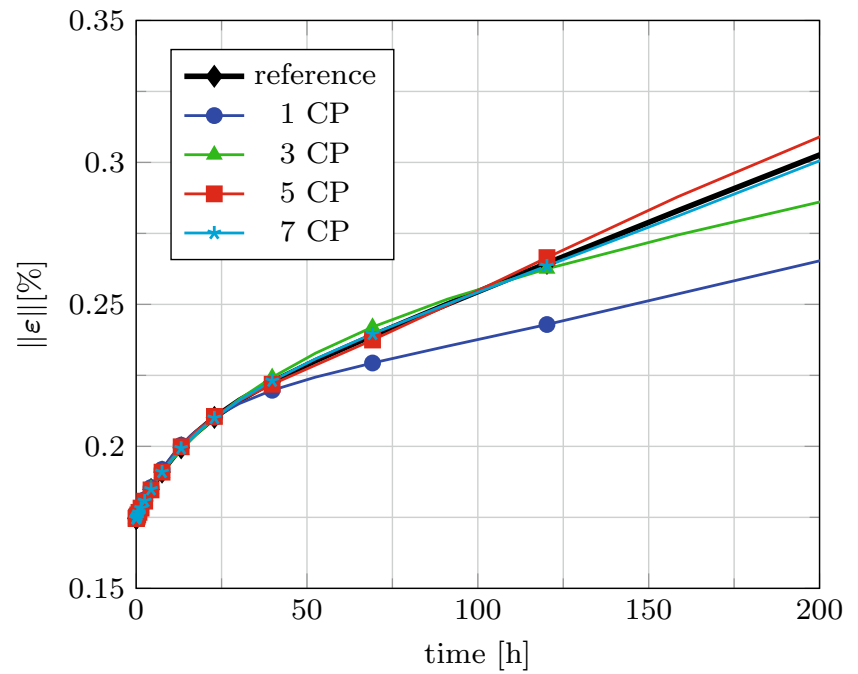

(a)

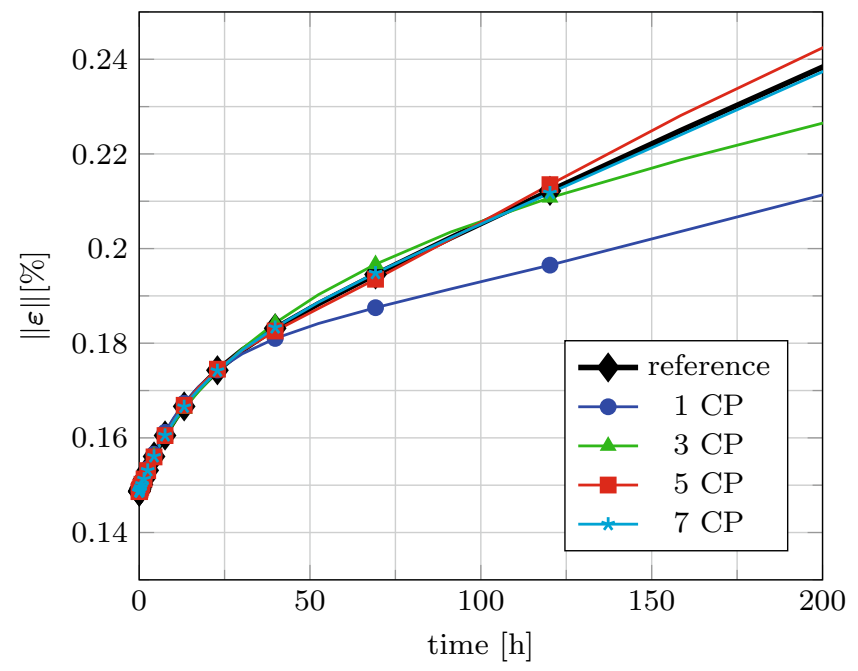

(c) where, for Burgers' model, the creep tensor has the form

$\mathbb{J}(x, t)=\mathbb{D}(x)+\mathbb{B}(x)\left(1-e^{t / \tau}\right)+\mathbb{F}(x) t$.

Consider, for some fixed $b>0$ and a positive integer $n$, the ansatz

$$
\begin{aligned}
J^{\text {coll }}(t) & :=\mathbb{D}^{\text {hom }}+\sum_{k=-n}^{n} \mathbb{B}_{k}^{\text {hom }}\left(1-e^{-b^{-k} t / \tau}\right) \\
& +\mathbb{F}^{\text {hom }} t
\end{aligned}
$$

where $\mathbb{D}^{\text {hom }}, \mathbb{F}^{\text {hom }}$ and $\mathbb{B}_{k}^{\text {hom }}$ are possibly anisotropic fourth-tensors to be determined. (28) encodes an instantaneous anisotropic elastic response via $\mathrm{D}^{\text {hom }}$, an anisotropic

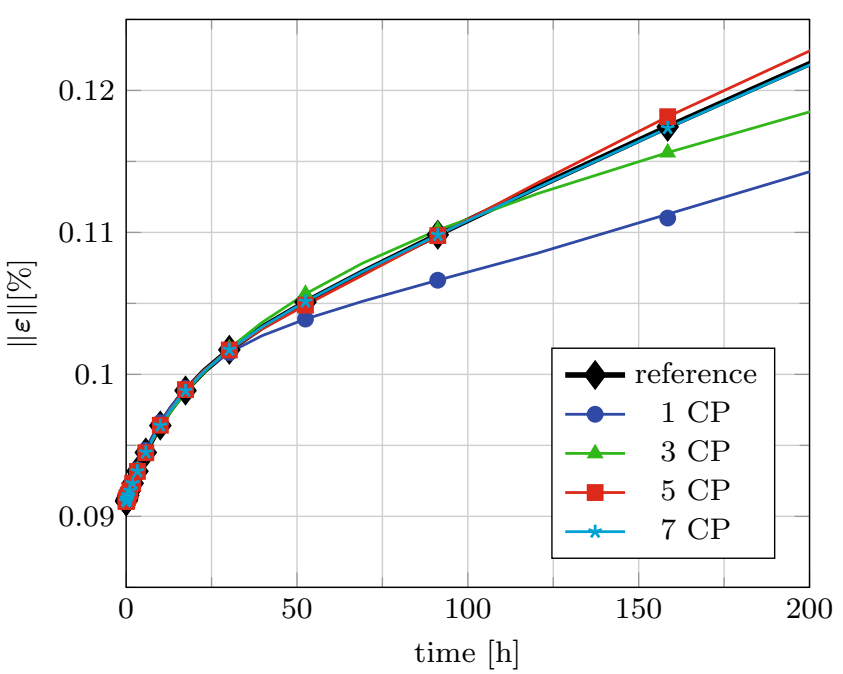

(b)

Fig. 10 Frobenius norm of the creep strain, in uniaxial creep tests in main fiber direction for different fiber orientations and number of collocation points. a Isotropic. b Unidirectional. c Planar isotropic 
Fig. 11 Maximal relative error of the strain in uniaxial creep simulations up to $10^{4} \mathrm{~h}$ for different numbers of collocation points, loading directions and fiber orientations

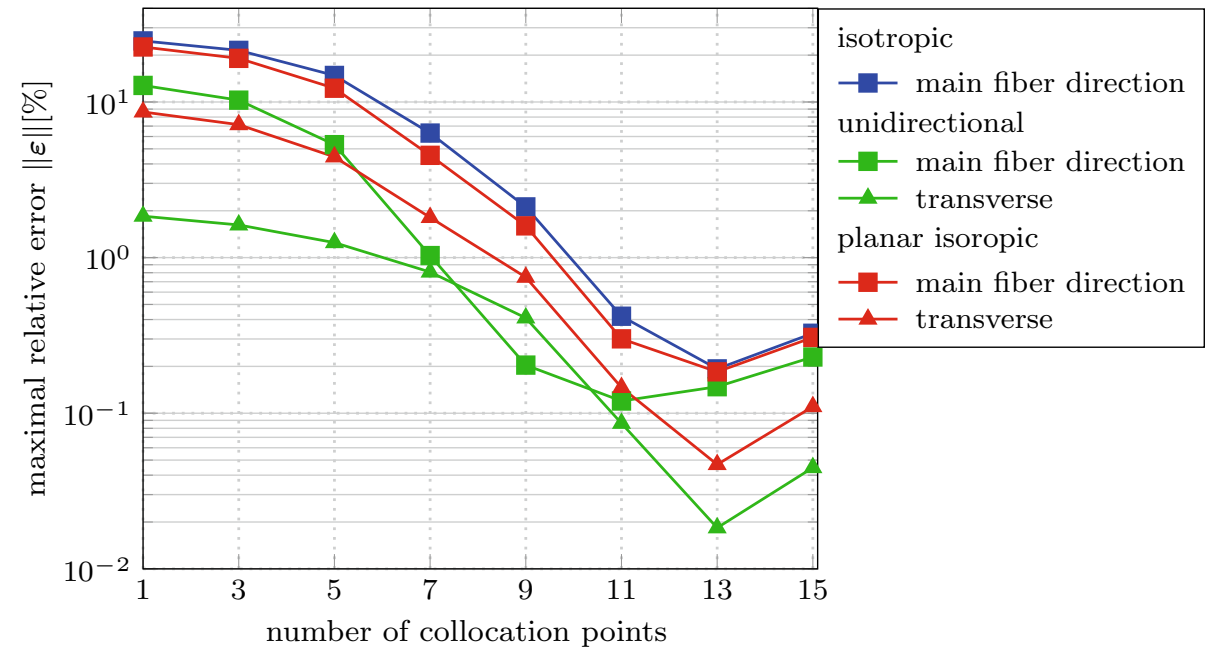

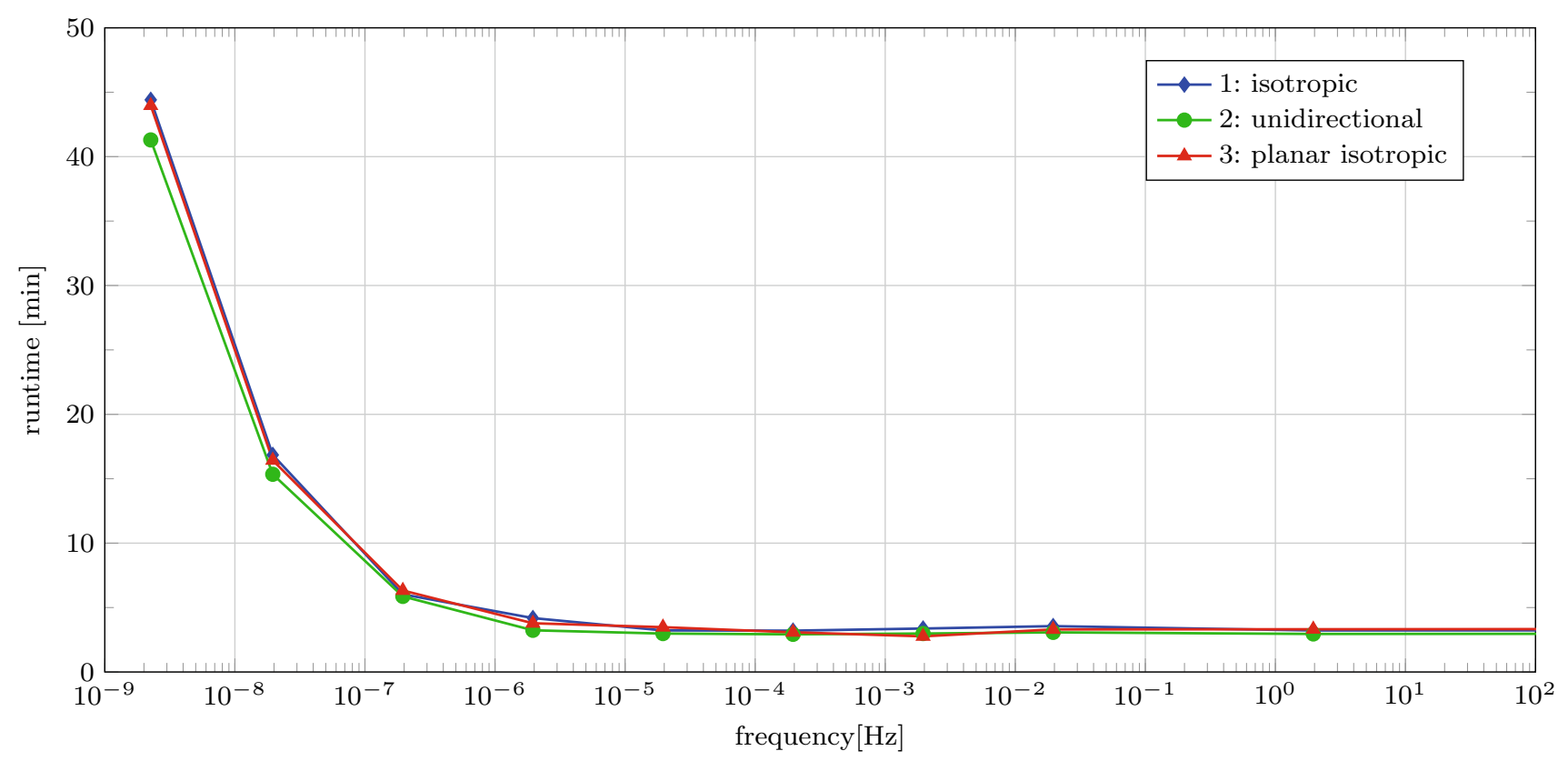

Fig. 12 Runtimes for the three extreme fiber orientations per collocation point

anisotropic fluidity $\mathbb{F}^{h o m}$, and a number of anisotropic relaxation tensors, connected to rescaled relaxation times $b^{k} \tau$, where $b$ serves as a logarithmic base, and $n$ limits the bandwidth of relaxation times. Schapery considered formally minimizing the total Frobenian square error

$\int_{0}^{t}\left\|\mathrm{~J}^{\text {coll }}(t)-\mathrm{J}^{\text {hom }}(t)\right\|^{2} d t \longrightarrow \min$

among all (28) for fixed $b$ and $n$. In a first step, $\mathbb{D}^{h o m}$ and $\mathbb{F}^{\text {hom }}$ are obtained by homogenizing $\mathbb{D}$ and $\mathbb{F}$, respectively, which correspond to the initial stiffness and the transient creep fluidity. Then, for $s_{l}=b^{-l} / \tau(l=-n, \ldots, n)$ the $2 n+1$ compliance fields
$\mathbb{D}(x)+\mathbb{B}(x) \frac{1}{1+s_{l} \tau}+s_{l}^{-1} \mathbb{F}(x)$

are homogenized, giving rise to homogenized tensors $\mathbb{K}_{l}^{\text {hom }}$. Finally, the tensors $\mathbb{B}_{k}^{\text {hom }}$ are eliminated from the $2 n+1$ tensor equations

$\mathbb{D}^{\text {hom }}+\sum_{k=-n}^{n} \mathbb{B}_{k}^{h o m} \frac{1}{1+s_{l} \tau b^{k}}+\mathbb{F}^{h o m} / s_{l}=\mathbb{K}_{l}$.

\subsection{On the number of collocation points for Schapery's method}

In this section we study the applicability of Schapery's method in our context. 


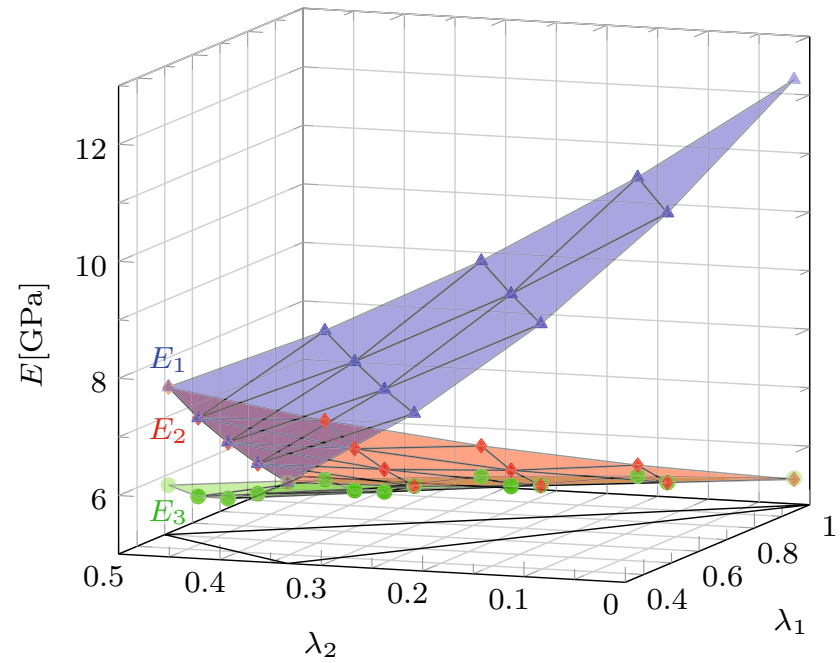

(a)

Fig. 13 Instantaneous orthotropic Young's moduli for different fiber orientations. a Young's modulus surface for the discretized fiber orientation triangle. b Young's moduli plotted along the corners of the fiber

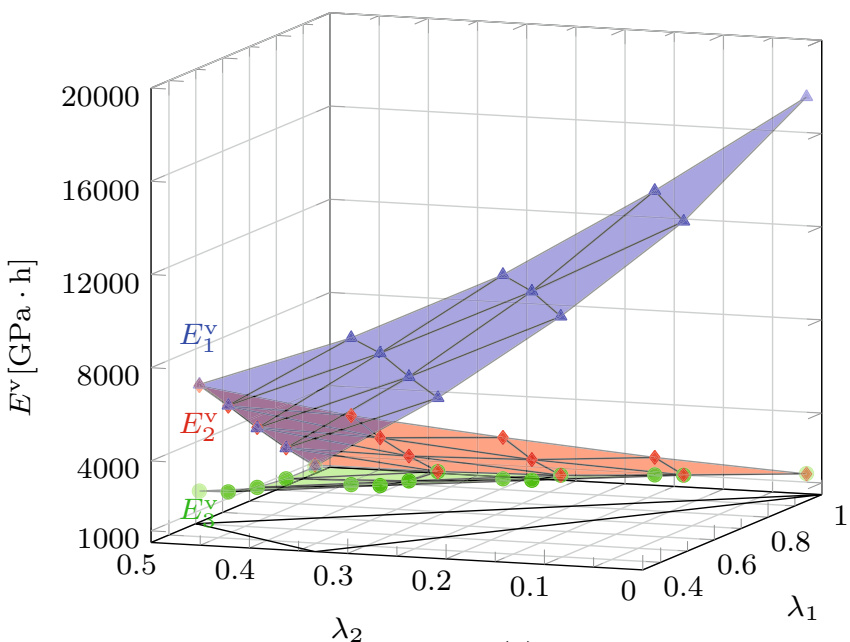

(a)

Fig. 14 Viscous orthotropic Young's moduli for different fiber orientations. a Young's modulus surface for the discretized fiber orientation triangle. b Young's moduli plotted along the corners of the fiber orienta-

With the previously established parameters, an RVE edge length of three times the fiber length and a resolution of $1 \mu \mathrm{m}$, we have computed solutions to the Schapery problems (30) for a different number of collocation points, ranging from 1 to 7 , and the basis $b=10$.

To assess the quality of Schapery's method for different collocation points, we have conducted a creep simulation with $1 \mathrm{MPa}$ stress applied in $x$-direction, with logarithmically distributed time steps up to $10^{4} \mathrm{~h}$. In Fig. 10 the creep strains computed from Schapery's model (28) are compared to the full resolution computation for the three extreme orientations.

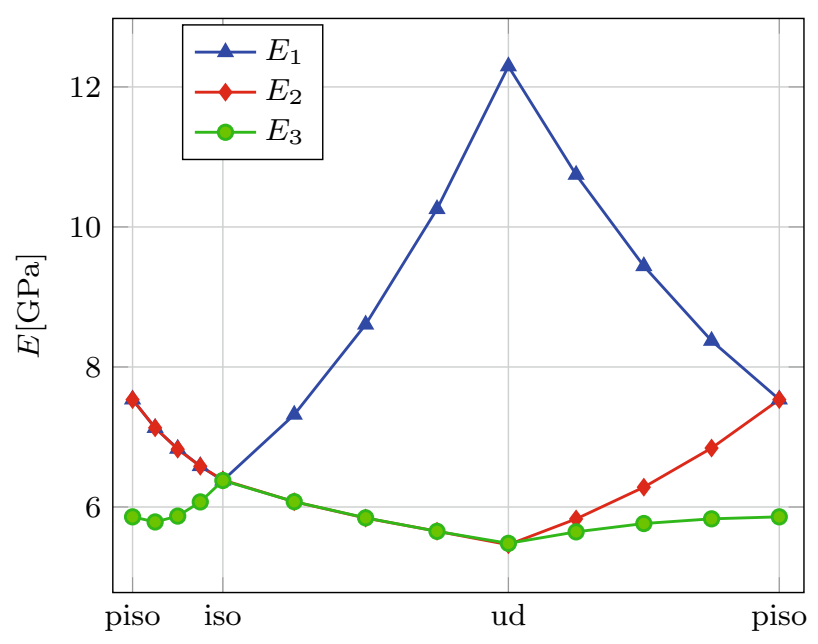

(b)

orientation triangle (piso, iso, ud corresponds to the planar isotropic, isotropic, unidirectional fiber orientation)

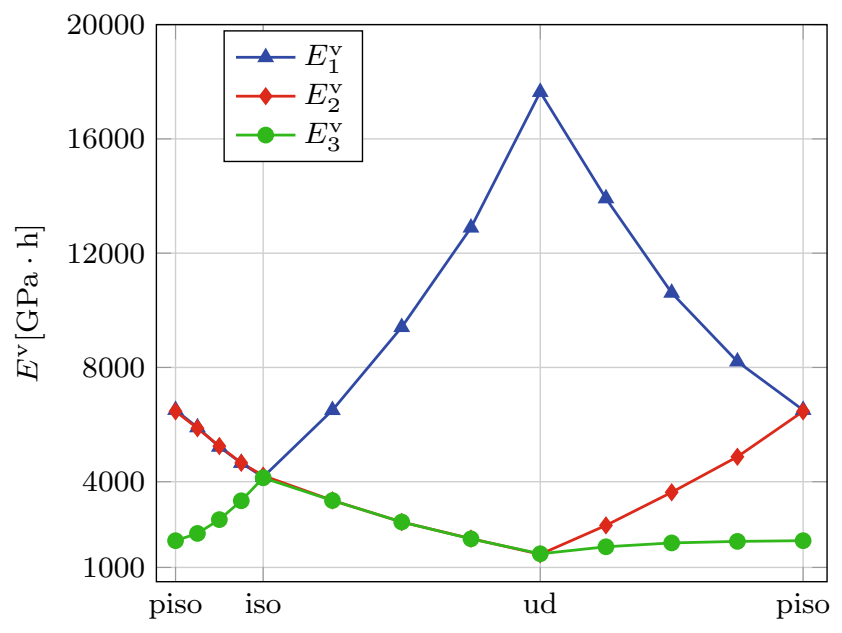

(b)

tion triangle (piso, iso, ud corresponds to the planar isotropic, isotropic, unidirectional fiber orientation)

For the isotropic fiber orientation state, see Fig. 10a, a single collocation point starts to strongly deviate from the reference curve at about $40 \mathrm{~h}$, whereas 3 collocation points start to deviate at about $120 \mathrm{~h} .5$ collocation points slightly overestimate the creep strain, whereas the stress-strain curve for 7 collocation points is hard to distinguish from the reference solution.

The behavior for the unidirectional and the planar isotropic geometries are similar to the isotropic case.

To gain further insight into the quality of the Schapery approximation we consider the maximal relative error in the strain for the different collocation methods, computed 


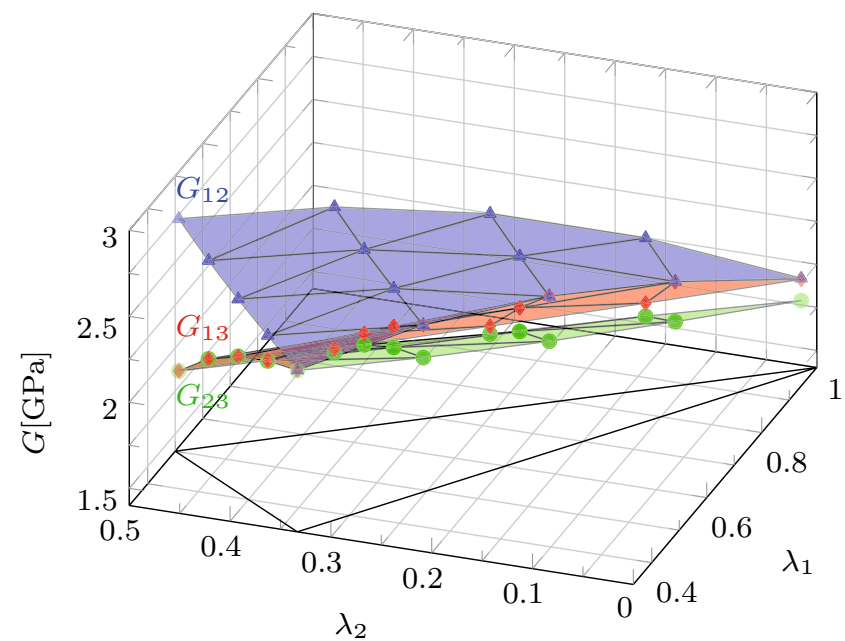

(a)

Fig. 15 Instantaneous orthotropic shear moduli for different fiber orientations. a Shear modulus surface for the discretized fiber orientation triangle. b Shear moduli plotted along the corners of the fiber orienta-

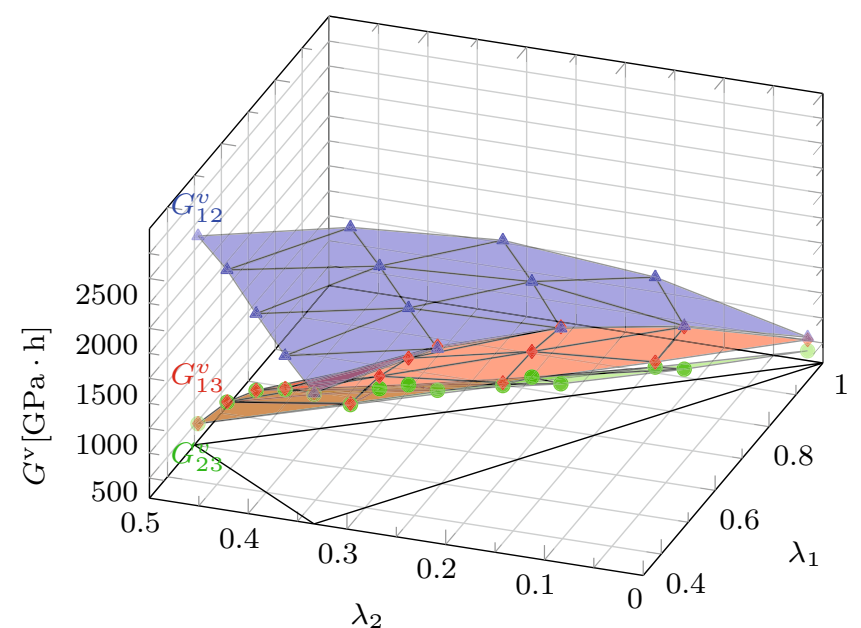

(a)

Fig. 16 Viscous orthotropic shear moduli for different fiber orientations. a Shear modulus surface for the discretized fiber orientation triangle. $\mathbf{b}$ Shear moduli plotted along the corners of the fiber orienta-

relative to the full-field solution. Here, maximal means the maximum error up to $10^{4} \mathrm{~h}$ of applied stress. Figure 11 shows that there are strong differences in the error, depending on the direction of applied stress and the microstructure. The relative error is smallest for the unidirectional structure and tension in transverse direction, remaining below $1 \%$ even for a single collocation point. This is not unexpected, as this loading scenarios is dominated by the matrix response, and the matrix is described by Burgers' model, which features only a single collocation point. The situation in transverse direction strongly contrasts to extension in fiber direction for the unidirectional structure. For up to 3 collocation points,

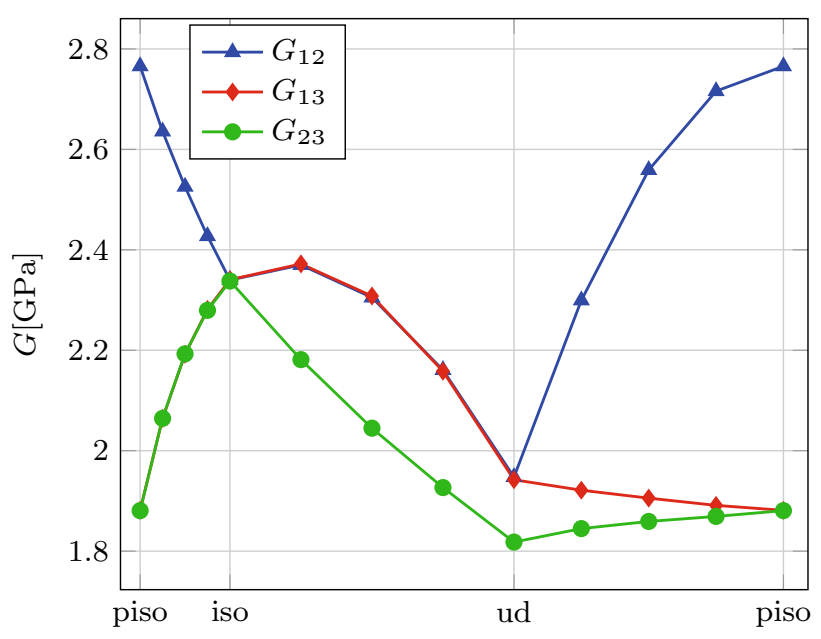

(b)

tion triangle (piso, iso, ud corresponds to the planar isotropic, isotropic, unidirectional fiber orientation)

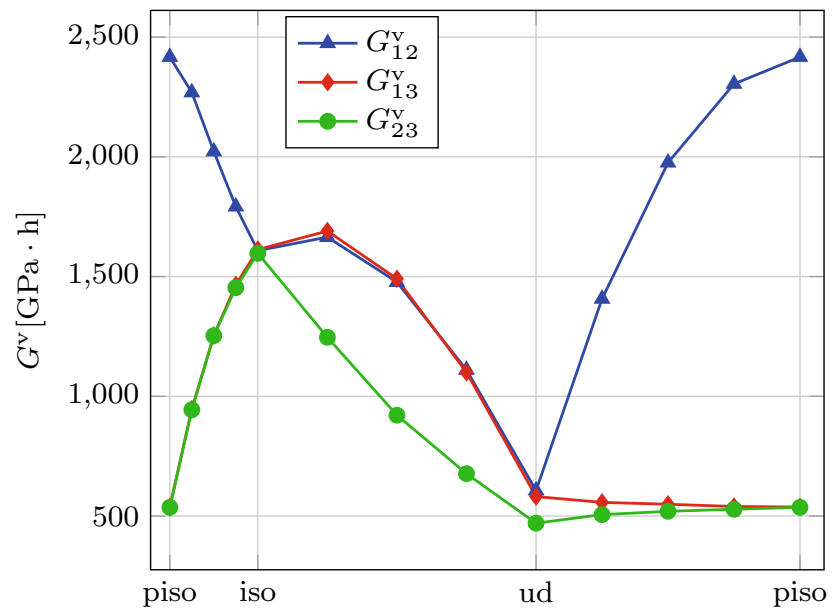

(b)

tion triangle (piso, iso, ud corresponds to the planar isotropic, isotropic, unidirectional fiber orientation)

the relative error is larger than $10 \%$, but decreases quickly for increased number of collocation points, and remains below $1 \%$ for seven collocation points. The behavior of the planar isotropic structure, loaded transversely, is somewhat similar to the unidirectional structure loaded in fiber direction. Also, there are similarities between the isotropic fiber orientation and the planar isotropic microstructure loaded in fiber direction: for these scenarios, the errors are largest, and decrease rather slowly. A relative error below $1 \%$ is reached for at least 9 collocation points. All in all we see that a relative error below $1 \%$ is guaranteed for more than 9 collocation points. 
rel. error [\%]

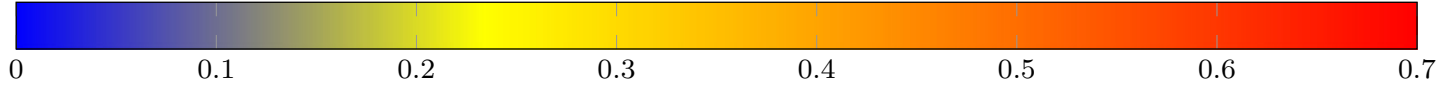

(a) $E_{1}$

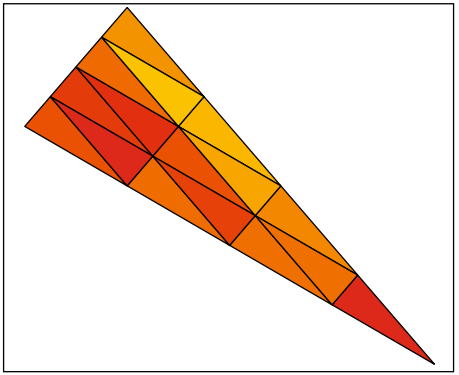

(d) $G_{23}$

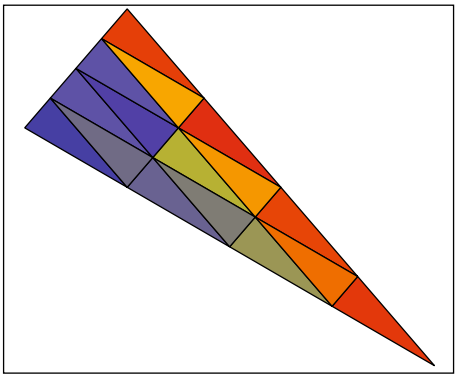

(g) $\nu_{32}$

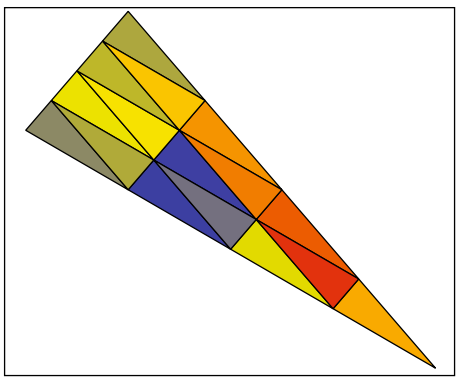

(b) $E_{2}$

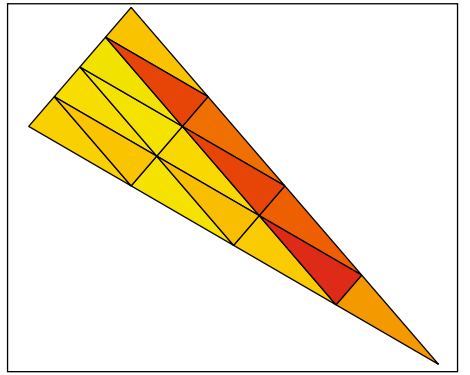

(e) $G_{13}$

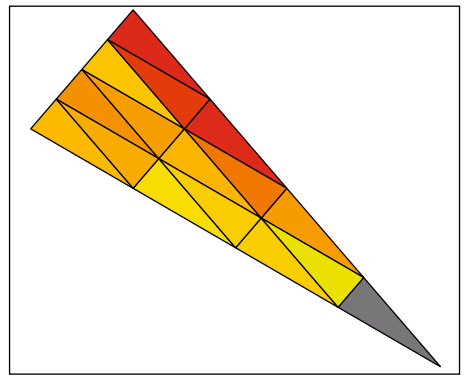

(h) $\nu_{31}$

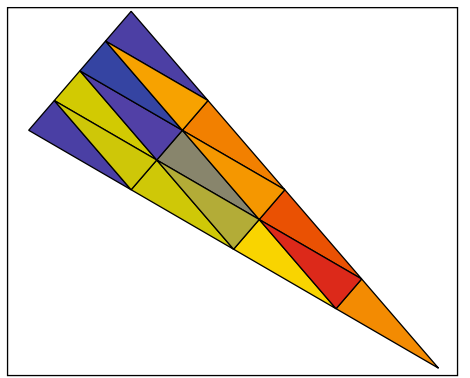

(c) $E_{3}$

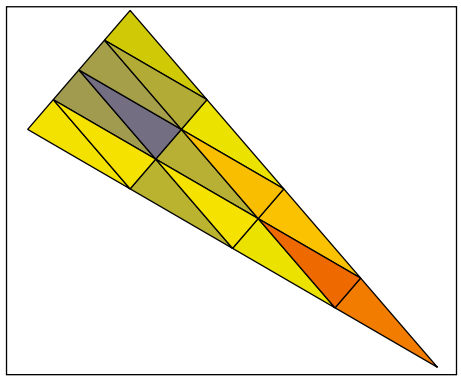

(f) $G_{12}$

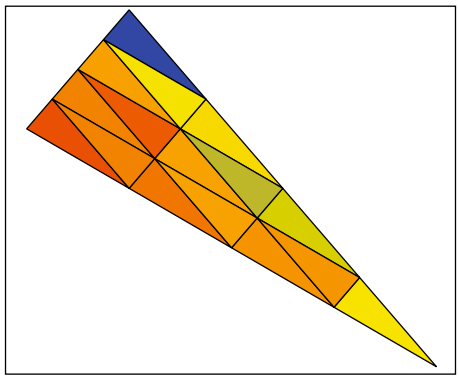

(i) $\nu_{21}$

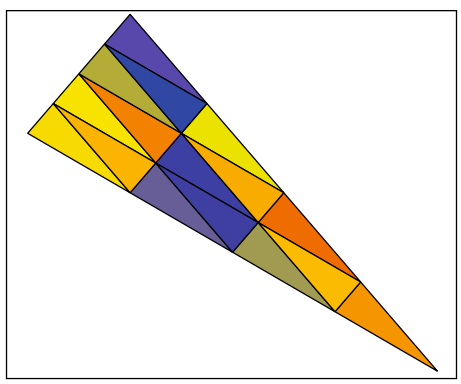

Fig. 17 Relative interpolation error of the instantaneous elastic orthotropic constants. a $E_{1}$. b $E_{2}$. c $E_{3}$. d $G_{23}$. e $G_{13}$. f $G_{12}$. g $\nu_{32}$. h $\nu_{31}$. i $\nu_{21}$

As a side remark notice that the error in Fig. 11 increases for more than 13 collocation points. This is no mistake, as these frequencies have little impact for creep up to $10^{4} \mathrm{~h}$, but change the optimum in the minimization (29) which covers also much larger times $t \in[0, \infty)$. To further increase the accuracy, the base $b$ would have to be changed from its current value 10 . However, for the problem at hand, we consider the accuracy to be sufficient.

Last but not least we take a look at the computational costs of the simulations, which were carried out on 64 MPI compute nodes, equipped with a dual Intel Xeon E5-2670 and 64 GB RAM, on our cluster. The runtimes are plotted in Fig. 12 as a function of the frequency $\tau b^{k}$ with $\tau=14.2 \mathrm{~h}$ and varying integers $k$, see Table 1 .
For $k=0$ a runtime of $3.08 \mathrm{~min}$ is required. Then, as $\mathrm{k}$ decreases, the time slightly increases up to the elastic case "at infinity" requiring $3.5 \mathrm{~min}$. For increasing $k$, the runtimes also increase compared to $k=0$, at about 4 min for $k=1$ and about $6 \min$ for $k=2$. For $k=4$, already about $43 \mathrm{~min}$ are necessary for resolving the unit cell problem. Thus, the viscous computations are the most time-demanding, confirming the findings of Sect. 3.4.

\subsection{Discussion of the results and errors}

The database was filled with the previously determined parameters, i.e. an RVE length of thrice the fiber length, a resolution of $1 \mu \mathrm{m}$ and 9 collocation points for Schapery's 
rel. error [\%]

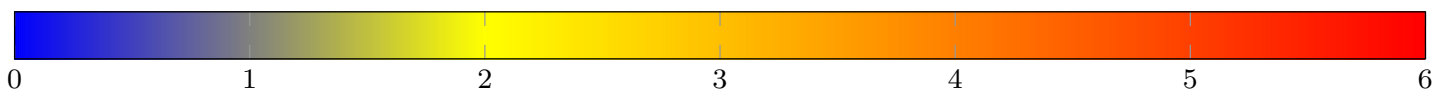

(a) $E_{1}^{\mathrm{v}}$

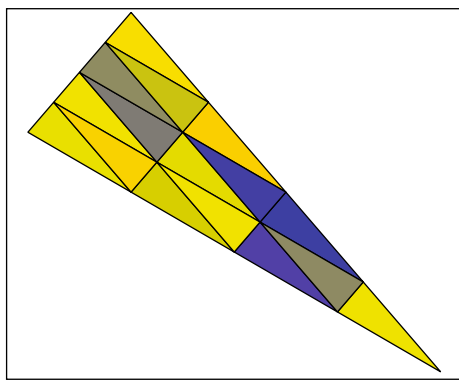

(d) $G_{23}^{\mathrm{v}}$

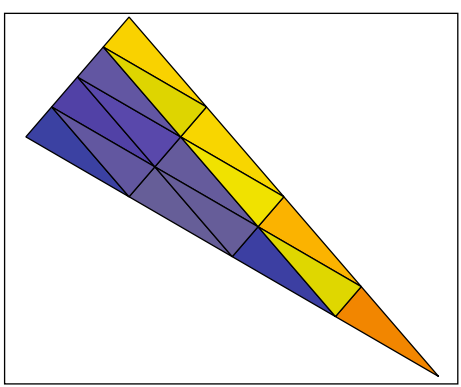

(g) $\nu_{32}^{\mathrm{v}}$

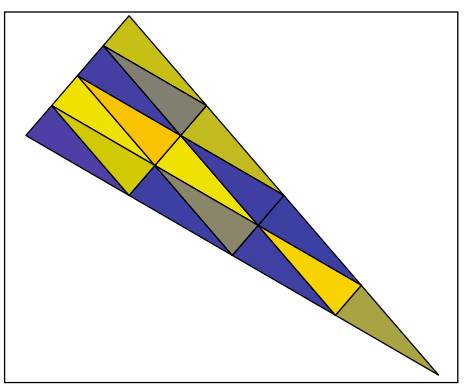

(b) $E_{2}^{\mathrm{v}}$

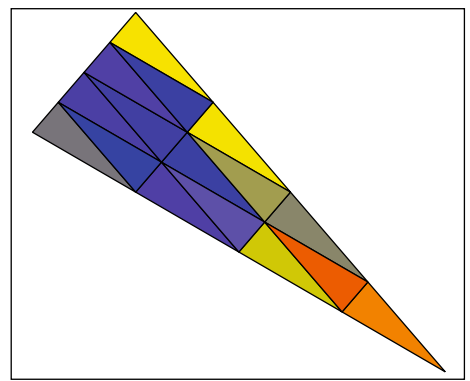

(e) $G_{13}^{\mathrm{v}}$

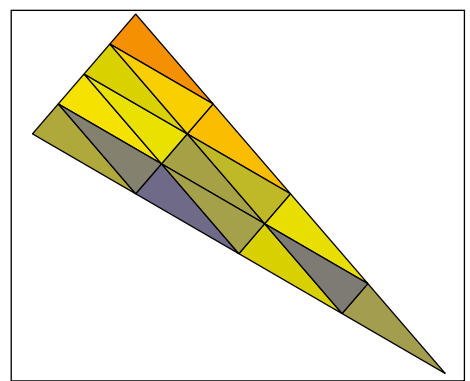

(h) $\nu_{31}^{\mathrm{v}}$

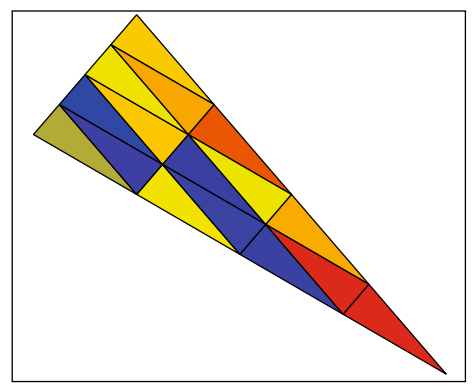

(c) $E_{3}^{\mathrm{v}}$

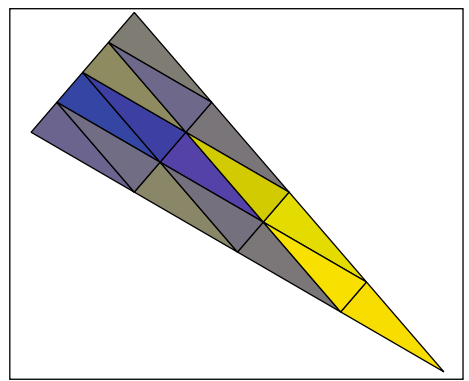

(f) $G_{12}^{\mathrm{v}}$

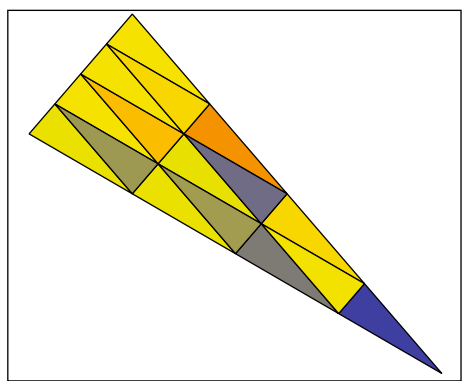

(i) $\nu_{21}^{\mathrm{v}}$

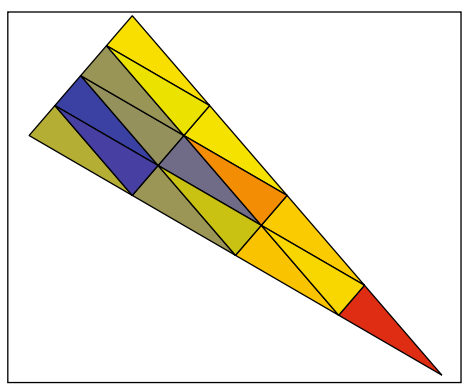

Fig. 18 Relative interpolation error of the viscous orthotropic constants. a $E_{1}^{v}$. b $E_{2}^{v}$. c $E_{3}^{v}$. d $G_{23}^{v}$. e $G_{13}^{v}$. f $G_{12}^{v}$. $\mathbf{g} v_{32}^{v}$. h $v_{31}^{v}$. i $v_{21}^{v}$

method, and a regular triangulation of the fiber orientation triangle with 15 nodes, as shown in Fig. 3. The entire computation took about $15 \mathrm{~h}$ on 64 MPI compute nodes, equipped with a dual Intel Xeon E5-2670 and 64 GB RAM, on our cluster.

As the prescribed fiber orientation tensors of fourth order are orthotropic, the effective tensors $\mathbb{K}_{l}$, and thus, the tensors $\mathbb{D}^{\text {hom }}, \mathbb{B}_{k}^{\text {hom }}$ and $\mathbb{F}^{\text {hom }}$ from (28) are orthotropic as well, see Schneider [44]. Hence it is reasonable to investigate the corresponding orthotropic engineering constants.

First, in Fig. 13, we take a look at the orthotropic Young's moduli of the instantaneous elastic response, i.e. those which can be read from $\mathbb{D}^{\text {hom }}$. Figure 13 a shows the three Young's moduli $E_{1}, E_{2}$ and $E_{3}$ as graphs plotted over the fiber ori- entation triangle, whereas Fig. 13b restricts the view to the edges of the fiber orientation triangle. The distances between ud (unidirectional), isotropic (iso) and planar isotropic (piso) on the $x$-axis of Fig. 13 correspond to the lengths of the edges connecting the corners of the fiber orientation triangle. We see that the graphs are smooth, but non-linear, and two of the three Young's moduli coincide on the edges connecting udiso and iso-piso, as they correspond to transversely isotropic fiber orientations. The contrasts between the Young's moduli is largest at the ud state (by about a factor of 2), much smaller at the piso state (about $\frac{4}{3}$ ), and of course, minimal for the isotropic state.

For comparison, the "viscous" Young's moduli, i.e. those which can be read from $\mathbb{F}^{\text {hom }}$, are plotted in Fig. 14. The qual- 
itative behavior of the graphs is similar to the instantaneous Young's moduli. However, the contrasts are much larger. Indeed, for the ud case, the Young's modulus in fiber direction $E_{1}^{v}$ and the transverse Young's modulus $E_{2}^{v}=E_{3}^{v}$ differ by a factor of roughly 18 , and the contrast for the piso case computes as about 5 . Thus, the creeping behavior of a composite exhibits a much higher anisotropy than the elastic response.

Next we investigate the instantaneous shear moduli $G_{12}$, $G_{13}$ and $G_{23}$ in Fig. 15. In this case, the differences between the different shear moduli is largest for the piso structure, with a contrast of about 1.5. Overall, the graphs appear to be at least quadratic.

Last but not least we take a look at the "viscous" shear moduli, which, as for the Young's moduli, exhibit qualitative similarities to their instantaneous counterparts, but differ strongly quantitatively. Indeed, the contrast between the shear moduli $G_{12}^{v}$ and $G_{13}^{v}=G_{23}^{v}$ at the piso state is about 5 (Fig. 16).

We have shown piecewise linear approximations of the orthotropic constants, considered as functions on the fiber orientation triangle. It remains to assess the quality of the approximation. For this purpose we have repeated the computation of effective elastic and viscous tensors $\mathbb{D}^{\text {hom }}$ and $\mathbb{F}^{\text {hom }}$ for the fiber orientations corresponding to the centers of mass of the triangles of our chosen triangulation and compared those tensors to their counterparts arising by interpolation. We investigate the relative error of the orthotropic elastic constants of the instantaneous elastic response in Fig. 17. The overall level of the relative error is rather small, bounded above by $0.7 \%$.

For the orthotropic constants of the viscous tensor $\mathbb{F}^{\text {hom }}$ the relative errors arising from interpolation are depicted in Fig. 18. The largest error of about $6 \%$ occurs for Poisson's ratio $v_{31}$. For the Young's moduli $E_{1}^{v}, E_{2}^{v}$ and $E_{3}^{v}$ the relative error is smaller than $2 \%$ except for the tip of the fiber orientation triangle and $E_{2}^{v}$, corresponding to the vicinity of the ud orientation, where the relative error rises to about $4 \%$. This results from the strong contrast between the in fiber Young's modulus and the transverse Young's moduli in the viscous case, see Fig. 15b. The relative errors for the shear moduli are bounded by $4 \%$.

All in all, the interpolation scheme is very accurate, except for some extreme cases (the Poisson's ratio in the viscous case in the vicinity of the ud state). Still, we consider the maximum error of about $6 \%$ acceptable since in most other cases, the relative errors are well below $5 \%$ relative error and thus within engineering accuracy.

\section{A computational example}

To prove our fiber orientation interpolation concept we investigate the viscoelastic creep behavior of a quick release
Table 4 Material parameters used for the injection molding simulation

\begin{tabular}{ll}
\hline Parameter & Value \\
\hline Density & $1410 \mathrm{~kg} / \mathrm{m}^{3}$ \\
Injection temperature & $275^{\circ} \mathrm{C}$ \\
Mold temperature & $40{ }^{\circ} \mathrm{C}$ \\
Specific heat & $2400 \mathrm{~J} / \mathrm{K}$ \\
Thermal conductivity & $0.25 \mathrm{~W} /(\mathrm{m} \mathrm{K})$ \\
Initial orientation & Isotropic \\
Fiber aspect ratio & $r_{a}=20$ \\
Folgar-Tucker coefficient & $C_{i}=0.01$ \\
Particle number & $N_{p}=0$ \\
Glass transition temperature & $T_{r e f}=230^{\circ} \mathrm{C}$ \\
$A_{0}$ & $0.1 \mathrm{~s}$ \\
$A_{1}$ & 0.65 \\
$A_{2}$ & $0.0211 / \mathrm{K}$ \\
$\eta_{0}$ & $100 \mathrm{Pas}$ \\
\hline
\end{tabular}

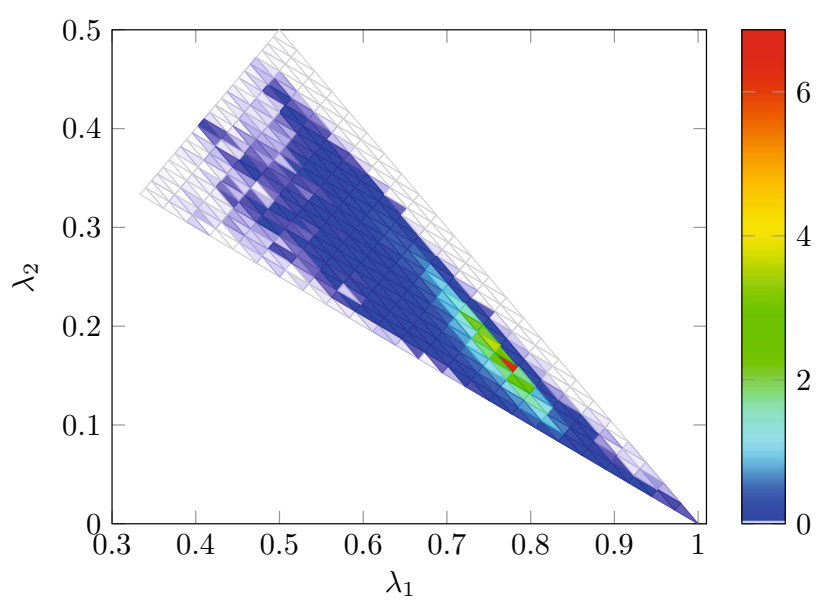

Fig. 19 Relative frequency of fiber orientations [\%]

buckle, whose CAD geometry is publicly available, ${ }^{2}$ see Fig. 1a

We conducted a mold filling simulation with injection molding software InjectionMoldingFoam [41], which is based upon OpenFOAM's incompressible two-phase flow solver [54]. Parameters for mold-filling release buckles are available in the literature, see Bhat et al. [8]. The parameters we used are summarized in Table 4 partially corresponding to the following variant of the Carreau-WLF equation [31]

$$
\eta(T, \dot{\gamma})=\eta_{0} \frac{e^{-A_{2}\left(T-T_{r e f}\right)}}{\left(1+\left(A_{0} \dot{\gamma}\right)^{2}\right)^{\frac{1-A_{1}}{2}}}
$$

\footnotetext{
${ }^{2}$ https://grabcad.com/library/quick-release-buckle-19mm.
} 


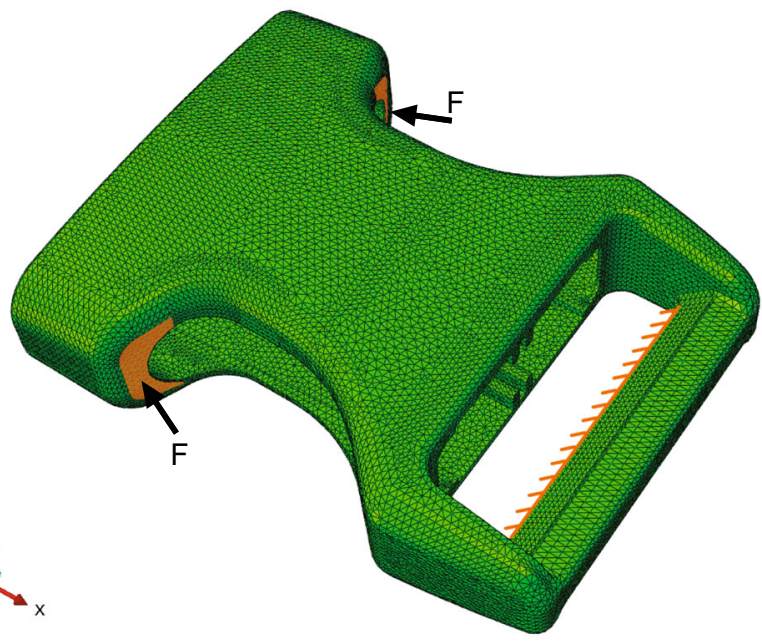

Fig. 20 Creep simulation boundary conditions with constant surface force $F$

and the injection points are shown in Fig. 1a. Notice that we do not include a back-coupling of the fiber orientation on the viscosity by setting the particle number $N_{p}$ to zero. However, we do include the temperature and shear rate dependence of the viscosity. For the simulation at hand, we use the ORW3 closure approximation [14].

The resulting fiber orientation is shown in Fig. 1 in a bottom and side view with the color scale of Fig. 2. An almost aligned state is dominant throughout the component, with more isotropic and planar states visible at the weld lines and the flat parts, respectively. A quantitative analysis of the fiber orientation, see Fig. 19, reveals that almost the full fiber orientation triangle is indeed covered by occurring fiber orientations. Only the vicinity of the isotropic state and a neighborhood of the line connecting the unidirectional and the planar isotropic state are left blank. However, most of the fiber orientations are concentrated around $\left(\lambda_{1}, \lambda_{2}\right)=$ (7.7, 0.15).

The calculated fiber orientation tensors serve as input for a component analysis using the commercial finite element software ABAQUS [1]. The mesh consists of approximately 310.000 linear tetrahedral elements (C3D4), see Fig. 20. A viscoelastic creep test up to $200 \mathrm{~h}$ was computed with a constant surface force $8 \mathrm{~N}$ applied as shown in Fig. 20, taking

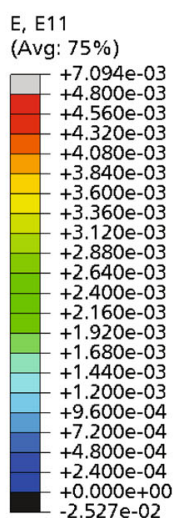

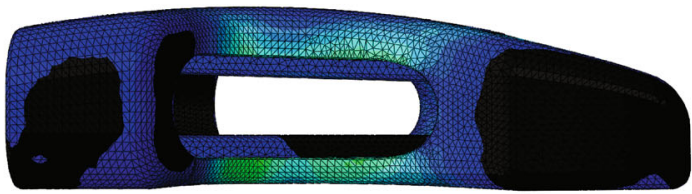

(a)

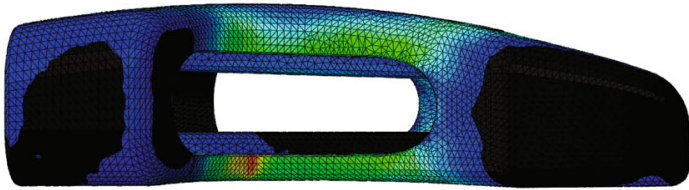

(c)

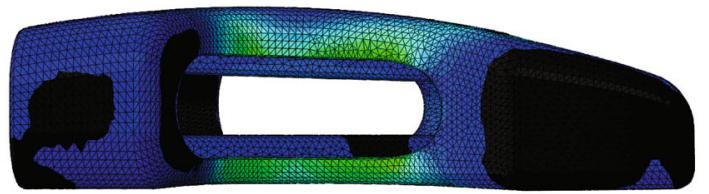

(b)

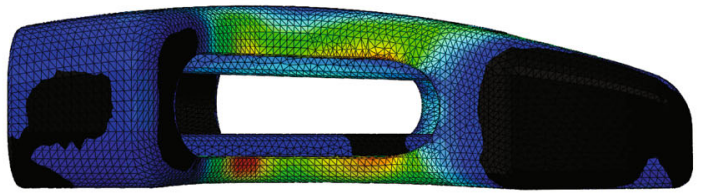

(d)

Fig. 21 Comparison of the strain in $x$-direction between the fiber orientation distribution obtained by injection molding simulation and an assumed isotropic fiber orientation. a Distributed orientation, $t=0 \mathrm{~h}$. b Isotropic, $t=0 \mathrm{~h}$. $\mathbf{c}$ Distributed orientation, $t=200 \mathrm{~h}$. d Isotropic, $t=200 \mathrm{~h}$

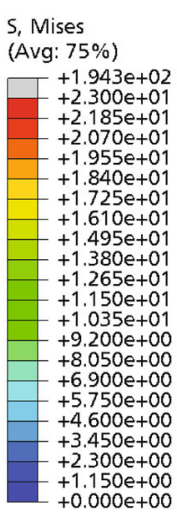

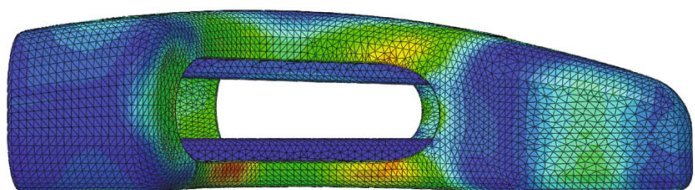

(a)

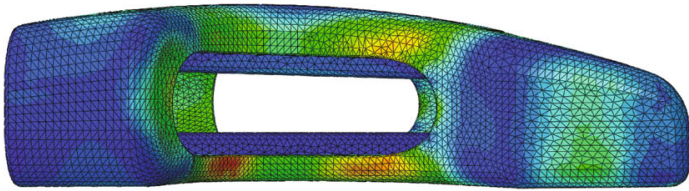

(c)

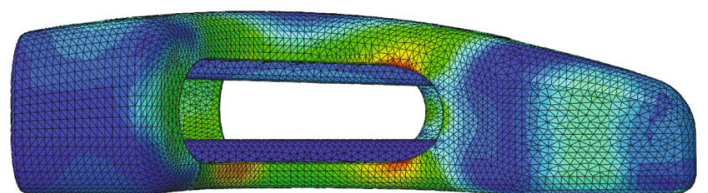

(b)

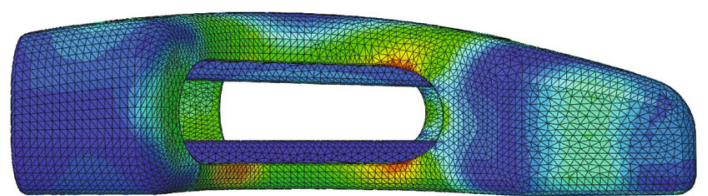

(d)

Fig. 22 Comparison of the von Mises stress between the fiber orientation distribution obtained by injection molding simulation and the isotropic fiber-filled component. a Distributed orientation, $t=0 \mathrm{~h}$. b Isotropic, $t=0 \mathrm{~h}$. c Distributed orientation, $t=200 \mathrm{~h}$. d Isotropic, $t=200 \mathrm{~h}$ 


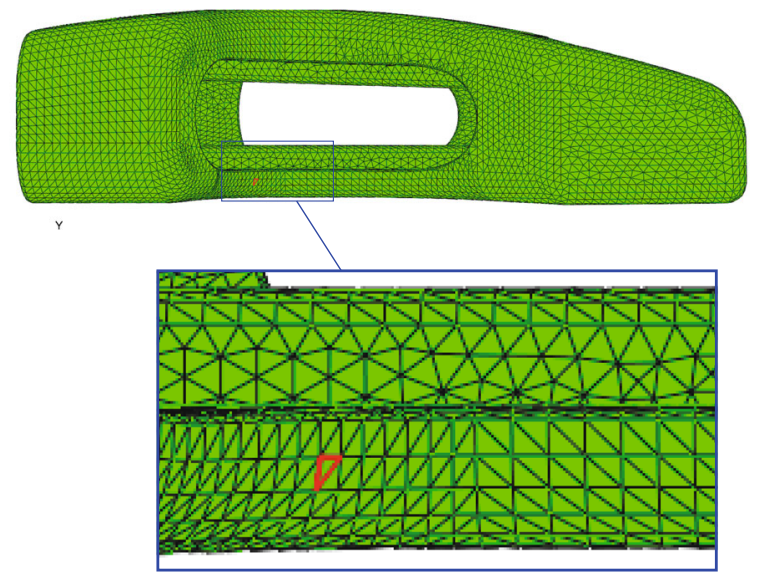

(a)

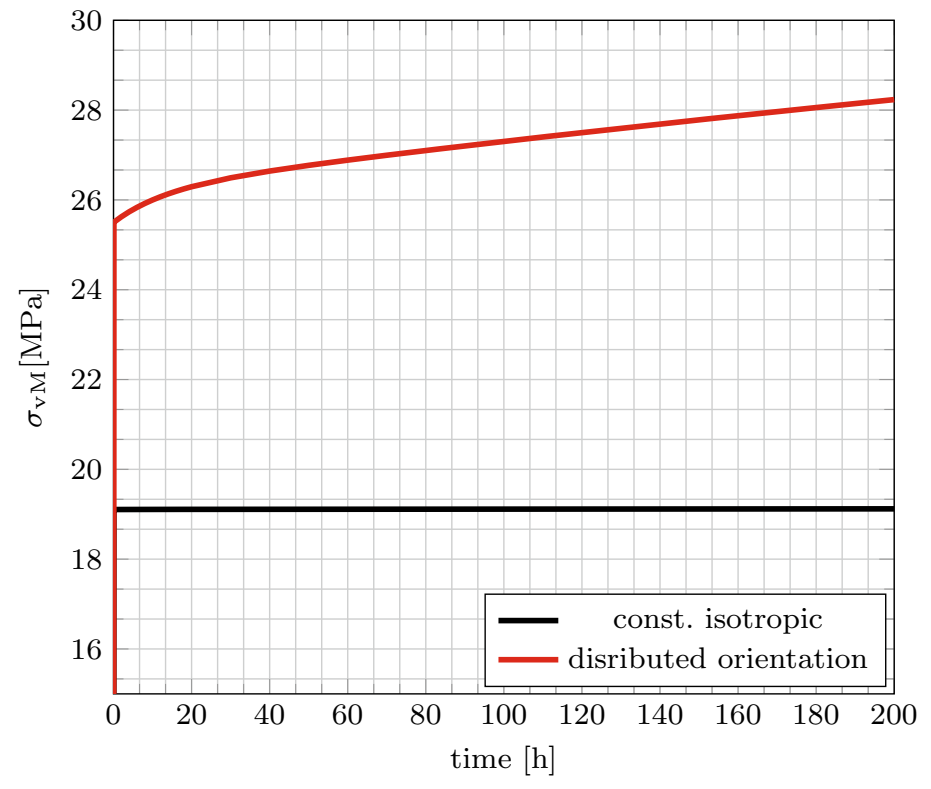

(b)

Fig. 23 Investigating the creep behavior in the most heavily loaded element. a Location of the element within the component. b Von Mises stress history

into account the identified viscoelastic database identified in Sect. 3. The computation comprised 30 time increments, spaced equidistantly on a logarithmic scale, taking about $2 \mathrm{~h}$ computation time on a desktop PC (single core). To assess the influence of the fiber orientation, for comparison, the computation was repeated for the same component, but where the fiber orientation was assumed isotropic throughout the whole geometry.

In Fig. 21 the strain in $x$-direction (cf. Fig. 20 for the axes) is shown, comparing the constant isotropic to the distributed fiber orientations. For the initial elastic response $(t=0)$, the strain distributions are even qualitatively similar. However, after $200 \mathrm{~h}$ strong differences in the strain fields can be observed. Indeed, for the isotropic fiber orientation spots with much higher strains are visible (in yellow and orange).

To further investigate this effect we show the corresponding von Mises equivalent stress in Fig. 22, and investigate the local relaxation behavior. For the constant isotropic component, the von Mises stress apparently changes only slightly during the loading history. For the distributed orientation, however, there is a load rebalancing comparing the stress fields at $t=0 \mathrm{~h}$ and $t=200 \mathrm{~h}$.

To gain deeper insight, we investigated the von Mises stress history up to $200 \mathrm{~h}$ in the most heavily loaded element for the distributed orientation, cf. Fig. 23. At the beginning of the loading, the stress level lies slightly above $25.5 \mathrm{MPa}$. After $200 \mathrm{~h}$, the stress level increased to about $28 \mathrm{MPa}$, i.e. by about $10 \%$.
We see that the constant isotropic computing overestimates the occurring local strains and does not account for the strongly anisotropic relaxation behavior of the component under consideration. In particular, an anisotropic component design necessitates larger safety factors than the computations with anisotropic relaxation behavior.

\section{Conclusion}

This work concerned the multiscale simulation of short fiber reinforced thermoplastics. Even though the fibers are much smaller than the typical component scale, the mechanical properties on the component scale are not homogeneous, but depend on the local fiber orientation. Thus, working with a single surrogate model for a fixed fiber orientation is insufficient, and the need to interpolate surrogate models corresponding to different fiber orientations arises.

We have proposed a versatile and robust approach to interpolate surrogate models, rigorously derived from the twopotential-framework $[23,25]$. The advantage of the approach is the ability to interpolate models of completely different type, in particular in terms of internal variables.

To test the framework on a non-trivial example, we investigated the viscoelastic creep behavior of PA66, reinforced by short glass fibers, and used Moulinec-Suquet's FFT-based computational homogenization scheme and Schapery's collocation method to identify anisotropic viscoelastic surrogate models for different fiber orientation states. It is shown that 
even for a rather rough triangulation of the fiber orientation triangle the interpolation errors are small.

Finally, the full identified viscoelastic model was used as a material model for an ABAQUS simulation of a benchmark component, exhibiting an anisotropy-induced strain rearrangement on the component scale.

This work contributes to bridging the gap between the insights provided by multiscale modelling of fiber reinforced composites achieved in recent years and fast computational analyses carried out by the constructing engineer. Indeed, even though the filling of the database is computationally demanding, the resulting material card enables extremely fast computations and is universal in the sense that it can be (re)used for different components built from the same material.

As already mentioned, the proposed fiber orientation interpolation scheme is not restricted to viscoelastic material behavior. The availability of surrogate models for different fiber orientations is the only prerequisite. In particular, viscoplastic or damage effects could be investigated. Furthermore, the interpolation scheme is not limited to problems at small strains, but extends effortlessly to the finite strain framework. As the interpolation is based on the free energies and the dissipation potentials, the question which strains and stresses should be interpolated does not arise but follows directly from the energetic formulation. Also, the scheme could be extended to incorporate local variations in fiber volume fraction.

Acknowledgements We are grateful to Matthias Kabel, Sarah Staub, Ralf Müller and Fabian Welschinger for fruitful discussions. Felix Ospald gratefully acknowledges financial support by the German Research Foundation (DFG), Federal Cluster of Excellence EXC 1075 "MERGE Technologies for Multifunctional Lightweight Structures".

Open Access This article is distributed under the terms of the Creative Commons Attribution 4.0 International License (http://creativecomm ons.org/licenses/by/4.0/), which permits unrestricted use, distribution, and reproduction in any medium, provided you give appropriate credit to the original author(s) and the source, provide a link to the Creative Commons license, and indicate if changes were made.

\section{References}

1. Abaqus (2014) Abaqus 6.14: analysis user's manual. Dassault Systemes Simulia Corp, Providence

2. Aboudi J (2004) The generalized method of cells and high-fidelity generalized method of cells micromechanical models-a review. Mech Adv Mater Struct 11(4-5):329-366

3. Advani SG, Tucker CL (1987) The use of tensors to describe and predict fiber orientation in short fiber composites. J Rheol 31(8):751-784

4. Andrä H, Kabel M, Staub S, Krizikalla F, Schulz V (2012) Numerische Homogenisierung für viskoelastische Faserverbundwerkstoffe. NAFEMS Online-Mag 21:70-83

5. Bakhvalov NS, Knyazev AV (1991) Efficient computation of averaged characteristics of composites of a periodic structure of essentially different materials. Sov Math Dokl 42(1):57-62
6. Bakhvalov NS, Panasenko GP (1989) Homogenization: averaging processes in periodic media. Kluwer, Dordrecht

7. Bella P, Otto F (2014) Corrector estimates for elliptic systems with random periodic coefficients. Multiscale Model Simul 14(4):14341462

8. Bhat HA, Subramaniam S, Pillai AK, L EK Elangovan M (2014) Analysis and design of mold for plastic side release Buckle using Moldflow software. Int J Res Eng Technol 3(5):366-372

9. Bhattacharjee S, Matouš K (2016) A nonlinear manifold-based reduced order model for multiscale analysis of heterogeneous hyperelastic materials. J Comput Phys 313:635-653

10. Bhattacharya K, Suquet P (2005) A model problem concerning recoverable strains of shape-memory polycrystals. Proc R Soc Lond A Math Phys Eng Sci 461(2061):2797-2816

11. Bhattacharya K, Suquet PM (2005) A model problem concerning recoverable strains of shape-memory polycrystals. Proc R Soc Lond A Math Phys Eng Sci 461(2061):2797-2816

12. Box G, Draper N (2007) Response surfaces, mixtures and ridge analyses, 2nd edn. Wiley, Hoboken

13. Camacho C, Tucker CL, Yalvac S, McGee R (1990) Stiffness and thermal expansion predictions for hybrid short fiber composites. Polym Compos 11:229-239

14. Chung DH, Kwon TH (2001) Improved model of orthotropic closure approximation for flow induced fiber orientation. Polym Compos 22(5):636-649

15. Doghri I, Tinel L (2005) Micromechanical modeling and computation of elasto-plastic materials reinforced with distributedorientation fibers. Int J Plast 21:1919-1940

16. Dvorak G (1992) Transformation field analysis of inelastic composite materials. Proc R Soc Lond A 4317:311-327

17. Engqvist EW, Lao B, Ren X, Vanden-Eijnden E W (2007) Heterogeneous multiscale methods: a review. Commun Comput Phys $2: 367-450$

18. FeelMath (2017) FeelMath. Fraunhofer Institute for Industrial Mathematics. http://www.itwm.fraunhofer.de/en/fraunhoferitwm.html

19. Findley W, Lai J, Onaran K (1989) Creep and relaxation of nonlinear viscoelastic materials: with an introduction to linear viscoelasticity. Dover, Mineola

20. Fritzen F, Leuschner M (2013) Reduced basis hybrid computational homogenization based on a mixed incremental formulation. Comput Methods Appl Mech Eng 260:143-154

21. Furukawa T, Yagawa G (1998) Implicit constitutive modelling for viscoplasticity using neural networks. Int J Numer Methods Eng 43(2):195-219

22. GeoDict (2017) GeoDict. Math2Market GmbH, Kaiserslautern, Germany. http://www.geodict.de. Accessed on 4 Jan 2017

23. Germain P, Nguyen QS, Suquet P (1983) Continuum thermodynamics. J Appl Mech 50:1010-1020

24. Haj-Ali R, Pecknold DA, Ghaboussi J, Voyiadjis GZ (2001) Simulated micromechanical models using artificial neural networks. J Eng Mech 127:730-738

25. Halphen B, Nguyen QS (1975) Sur les matériaux standard généralisés. J Méc 14:39-63

26. Hashin Z (1965) Viscoelastic behavior of heterogeneous media. J Appl Mech Trans ASME 32:630-636

27. Hashin Z (1970) Complex modulis of viscoelastic compositesI. General theory and application to particulate composites. Int $\mathbf{J}$ Solids Struct 6:539-552

28. Hill R (1963) Elastic properties of reinforced solids: some theoretical principles. J Mech Phys Solids 11:357-372

29. Jack DA, Smith DE (2005) An invariant based fitted closure of the sixth-order orientation tensor for modeling short-fiber suspensions. J Rheol 49(5):1091-1115 
30. Janovsky V, Shaw MKWS, Whiteman JR (1995) Numerical methods for treating problems of viscoelastic isotropic solid deformation. J Comput Appl Math 63(1-3):91-107

31. Kennedy PK (2013) Flow analysis of injection molds, 2nd edn. Hanser, Munich

32. Lai J, Bakker A (1996) 3-D Schapery representation for non-linear viscoelasticity and finite element implementation. Comput Mech 18(3):182-191

33. Le BA, Yvonnet J, He QC (2015) Computational homogenization of nonlinear elastic materials using neural networks. Int J Numer Methods Eng 104:1061-1084

34. Lefik M, Schrefler B (2003) Artificial neural network as an incremental non-linear constitutive model for a finite element code. Comput Methods Appl Mech Eng 192:3265-3283

35. Matouš K, Geers MGD, Kouznetsova VG, Gillman A (2017) A review of predictive nonlinear theories for multiscale modeling of heterogeneous materials. J Comput Phys 330:192-220

36. Michel J, Suquet P (2003) Nonuniform transformation field analysis. Int J Solids Struct 40(25):6937-6955

37. Montgomery-Smith S, He W, Jack DA, Smith DE (2011) Exact tensor closures for the three-dimensional Jeffery's equation. J Fluid Mech 680:321-335

38. Mosby M, Matouš K (2016) Computational homogenization at extreme scales. Extreme Mech Lett 6:68-74

39. Moulinec H, Suquet P (1994) A fast numerical method for computing the linear and nonlinear mechanical properties of composites. Comptes rendus de l'Académie des sciences Série II, Mécanique, physique, chimie, astronomie 318(11):1417-1423

40. Moulinec H, Suquet P (1998) A numerical method for computing the overall response of nonlinear composites with complex microstructure. Comput Methods Appl Mech Eng 157:69-94

41. Ospald F (2014) Numerical simulation of injection molding using OpenFOAM. Proc Appl Math Mech (PAMM) 14(1):673-674

42. Python (2017) Python language reference, version 2.7. Python Software Foundation, Wilmington

43. Schapery RA (1962) Approximate methods of transform inversion in viscoelastic stress analysis. In: Proceedings of the 4th US national congress on applied mechanics, vol 2, pp 1075-1085

44. Schneider M (2017) The sequential addition and migration method to generate representative volume elements for the homogenization of short fiber reinforced plastics. Comput Mech 59(2):247-263

45. Schneider M, Ospald F, Kabel M (2016) Computational homogenization on a staggered grid. Int J Numer Methods Eng 105(9):697720

46. Steiner K, für Techno-und Wirtschaftsmathematik FI (2012) MISES-FOK: Multiskalenintegrierende Struktureigenschaftssimulation der Faserorientierung für faserverstärkte Kunststoffe im Automobil- und Flugzeugbau: Abschlussbericht zum Forschungsvorhaben 03x0513F im BMBF-Rahmenprogramm WING : Bearbeitungszeitraum vom 01.03.2007 bis 31.12.2011
47. Suquet $P$ (1985) Local and global aspects in the mathematical theory of Plasticity. In: Sawchuk A, Bianchi G (eds) Plasticity today: modelling, methods and applications. Elsevier, London, pp 279310

48. Suquet P (1987) Elements of homogenization for inelastic solid mechanics. In: Sanchez-Palencia E, Zaoui A (eds) Homogenization techniques for composite media, vol 272. Lecture notes in physics. Springer, New York, pp 193-278

49. Takano N, Zako M, Ohnishi Y (1996) Macro-micro uncoupled homogenization procedure for microscopic nonlinear behavior analysis of composites. Mater Sci Res Int 2(2):81-86

50. Temizer I, Wriggers P (2007) An adaptive method for homogenization in orthotropic nonlinear elasticity. Comput Methods Appl Mech Eng 35-36:3409-3423

51. Terada K, Kikuchi N (1995) Nonlinear homogenization method for practical applications. ASME Appl Mech Div Publ AMD 212:1-16

52. Unger JF, Könke C (2008) Coupling of scales in multiscale simulation using neural network. Comput Struct 86:1994-2003

53. Ward IM (2013) Mechanical properties of solid polymers, 3rd edn. Wiley, Hoboken

54. Weller HG, Tabor HJG, Fureby C (1998) A tensorial approach to computational continuum mechanics using object-oriented techniques. Comput Phys 12(6):620-631

55. Wirtz D, Karajan N, Haasdonk B (2015) Surrogate modeling of multiscale models using kernel methods. Int $\mathrm{J}$ Numer Methods Eng 101:1-28

56. Woldekidan M (2011) Response modelling of bitumen, bituminous mastic and mortar. Ph.D. thesis, Delft University of Technology

57. Yang JL, Zhang Z, Schlarb AK, Friedrich K (2006) On the characterization of tensile creep resistance of polyamide 66 nanocomposites. Part I. Experimental results and general discussions. Polymer 47:2791-2801

58. Yang JL, Zhang Z, Schlarb AK, Friedrich K (2006) On the characterization of tensile creep resistance of polyamide 66 nanocomposites. Part II. Modeling and prediction of long-term performance. Polymer 47:6745-6758

59. Yvonnet J, He QC (2007) The reduced model multiscale method (R3M) for the non-linear homogenization of hyperelastic media at finite strains. J Comput Phys 223:341-368

60. Yvonnet J, Monteiro E, He Q (2013) Computational homogenization method and reduced database model for hyperelastic heterogeneous structures. Int J Multiscale Comput Eng 11:201225

61. Zaoui A (2002) Continuum micromechanics: survey. J Eng Mech 128:808-816

62. Zeman J, Vondřejc J, Novak J, Marek I (2010) Accelerating a FFTbased solver for numerical homogenization of periodic media by conjugate gradients. J Comput Phys 229(21):8065-8071

Publisher's Note Springer Nature remains neutral with regard to jurisdictional claims in published maps and institutional affiliations. 